\title{
Wave packet construction in two-dimensional quantum billiards: Blueprints for the square, equilateral triangle, and circular cases
}

\author{
M. A. Doncheski \\ Department of Physics, The Pennsylvania State University, Mont Alto, Pennsylvania 1723 t*
}

\author{
S. Heppelmann, R. W. Robinett, and D. C. Tussey \\ Department of Physics, The Pennsylvania State University, \\ University Park, Pennsylvania 1680d
}

\begin{abstract}
We present quasi-analytical and numerical calculations of Gaussian wave packet solutions of the Schrödinger equation for two-dimensional infinite well and quantum billiard problems with equilateral triangle, square, and circular footprints. These cases correspond to $N=3, N=4$, and $N \rightarrow \infty$ regular polygonal billiards and infinite wells, respectively. In each case the energy eigenvalues and wavefunctions are given in terms of familiar special functions. For the first two systems, we obtain closed form expressions for the expansion coefficients for localized Gaussian wavepackets in terms of the eigenstates of the particular geometry. For the circular case, we discuss numerical approaches. We use these results to discuss the short-time, quasi-classical evolution in these geometries and the structure of wave packet revivals. We also show how related half-well problems can be easily solved in each of the three cases.
\end{abstract}




\section{INTRODUCTION}

The use of wave packets to analyze the dynamics of quantum mechanical systems is an increasingly important aspect of the study of the classical-quantum interface. $\frac{1}{\text { Popular }}$ software packages ${ }^{2}$ can help students visualize the evolution of quantum states (in contrast to the more typical time-independent stationary state solutions seen in most textbooks) by allowing students to change parameters (such as the initial width of a wave packet). Such visualizations are potentially important because recent studies,,$^{3.4}$ have suggested that student understanding of time-development in quantum mechanics is rather limited.

Several authors have discussed pedagogical descriptions of various one-dimensional (1D) quantum mechanical problems using a wave packet approach, studying such topics as trans-

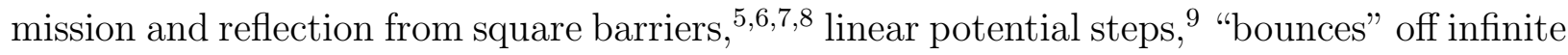
walls,,$\frac{10.11}{2}$ and bound state wave packets in single square wells in either position space $\frac{12}{2}$ or

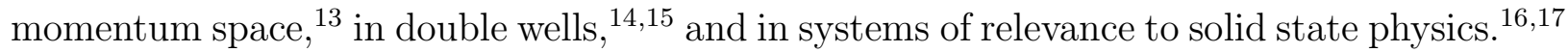
More recently, wave packet revivals have been studied in the context of familiar 1D quantum mechanical bound state systems such as 1D infinite wells and the "quantum bouncer"

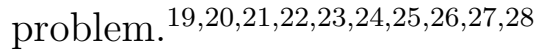

An obvious extension would be the study of wave packet propagation in two-dimensional infinite wells of various shapes (quantum billiards). In this paper we will discuss the construction of Gaussian-like wave packet solutions in three simple two-dimensional (2D) geometries, namely, the square, equilateral triangle, and circular infinite wells. We note that measurements of conductance fluctuations in ballistic microstructures 29 have been tentatively used to identify features in the power spectrum with particular closed orbits in a circular and stadium billiard. More recently, the realization of billiards ${ }^{30}$ with ultracold atoms in arbitrarily shaped 2D boundaries confined by optical dipole potentials has allowed the study of various chaotic and integrable shapes such as the stadium, ellipse, and circle.

The study of the energy eigenvalues and eigenfunctions in general 2D billiard systems can be used to probe quantum chaos, but in the cases we discuss here, the corresponding integrable classical motions are easily obtained and can be compared to, and contrasted against, the short-time quantum development. In each case, we make use of closed form solutions, and for the first two systems we show how to obtain simple expressions for the expansion coefficients of a gaussian wavepacket in terms of the eigenstates of the particular 
system; for the circular case we illustrate numerical results. We then use our results to discuss the short-time propagation of wave packets, compare them to classical orbits, and investigate the structure of longer-time quantum revivals.

The infinite square well provides an easy introduction to the subject, and the cases of the circular ${ }^{31}$ and equilateral triangle ${ }^{32}$ quantum billiards have recently been examined in the research literature. We will make appropriate use of the results in Refs. 31 and 32, but our discussions will focus on more pedagogical aspects, including other special cases of 2D billiards obtained by "foldings" of these cases to make connections to the degeneracy of the energy eigenvalues and the symmetry structure of energy eigenstates in simple geometries. The comparison of these three exactly soluble 2D infinite well problems and their quantum dynamics is a major thrust of this paper. Note that these systems correspond to $N=4$, $N=3$, and $N \rightarrow \infty$ regular polygonal billiard footprints, and it is an interesting open question how the exact quantum revivals we find for the $N=3$ and 4 cases change to the approximate ones for the $N \rightarrow \infty$ (circular) case.

We begin in Sec. II by reviewing the basic properties of Gaussian wave packets and the general time-dependence of $1 \mathrm{D}$ bound state systems, before discussing the $1 \mathrm{D}$ infinite well in Sec. III. In Sec. IV we consider the general time-dependence of systems with two quantum numbers, as well as the square billiard. We will also be able to use this geometry to examine the special case of the $45^{\circ}-45^{\circ}-90^{\circ}$ triangular billiard obtained from the square well by folding along a diagonal. In Sec. V we briefly review the properties of the quantum solutions to the Schrödinger equation in an equilateral triangle $\left(60^{\circ}-60^{\circ}-60^{\circ}\right)$ infinite well (one that is not frequently discussed in the pedagogical literature) and discuss both the short-time quasiclassical propagation of wave packets as well as derive the exact quantum revivals that are present in this system. We also note that half-well solutions for the $30^{\circ}-60^{\circ}-90^{\circ}$ triangle are easily obtained by folding along an axis of symmetry. Finally, we turn our attention in Sec. VI to the case of the circular billiard and again discuss how information on both the classical periodicities and possible quantum revivals is contained in the energy eigenvalue spectrum. In Sec. VII we present conclusions and some avenues for future investigation.

Although this work will focus mostly on the formalism required to construct Gaussian wave packets in these three geometries in the most efficient ways and will be restricted to static images of position-space probability densities, we have also produced animations of the short- and long-time development of such wave packet solutions in both 
the $1 \mathrm{D}$ and 2D infinite wells, illustrating the short-time, quasi-classical propagation and the structure of quantum revivals (and fractional revivals). These animations are available at 〈http://www.phys.psu.edu/〜rick/QM/qm.html〉 and include visualizations of the position- and momentum space probability densities, quantum expectation values compared to classical trajectories, and plots of the autocorrelation function for several different initial conditions for 1D and 2D Gaussian wave packets.

\section{ONE-DIMENSIONAL BACKGROUND}

\section{A. Gaussian wave packets}

For each of the geometries we consider, we will use initial Gaussian wave packets corresponding to standard free particle solutions of the Schrödinger equation. Although such solutions do not explicitly satisfy the boundary conditions at the wall(s), as long as they are sufficiently far from any infinite wall boundary, the resulting error can be made exponentially small, and the solutions can be reliably expanded in eigenstates of the billiard system. The initial wave forms we will use in one dimension are given by

$$
\langle p \mid G\rangle=\phi_{G}(p, 0)=\frac{\alpha^{1 / 2}}{\pi^{1 / 4}} e^{-\alpha^{2}\left(p-p_{0}\right)^{2} / 2} e^{-i p x_{0} / \hbar}
$$

which corresponds to

$$
\langle x \mid G\rangle=\psi_{G}(x, 0)=\frac{1}{b^{1 / 2} \pi^{1 / 4}} e^{-\left(x-x_{0}\right)^{2} / 2 b^{2}} e^{i p_{0}\left(x-x_{0}\right) / \hbar}
$$

where $b \equiv \alpha \hbar$. These two equivalent representations of the same Hilbert state vector, $|G\rangle$, are, of course, related by the Fourier transform

$$
\psi_{G}(x, 0)=\frac{1}{\sqrt{2 \pi \hbar}} \int_{-\infty}^{+\infty} \phi_{G}(p, 0) e^{i p x / \hbar} d p
$$

The initial expectation values for this general state are given by

$$
\langle x\rangle_{0}=x_{0}, \quad\left\langle x^{2}\right\rangle_{0}=x_{0}^{2}+\frac{b^{2}}{2}, \quad \Delta x_{0}=\frac{b}{\sqrt{2}},
$$

and

$$
\langle p\rangle_{0}=p_{0}, \quad\left\langle p^{2}\right\rangle_{0}=p_{0}^{2}+\frac{\hbar^{2}}{2 b^{2}}, \quad \Delta p_{0}=\frac{\hbar}{\sqrt{2} b}=\frac{1}{\sqrt{2} \alpha}
$$


We can easily vary the initial position $x_{0}$, momentum $p_{0}$, and width $\Delta x_{0}$ of the packet. The expectation value for the total (kinetic) energy is given by

$$
\langle\hat{E}\rangle=\frac{1}{2 \mu}\left\langle p^{2}\right\rangle=\frac{1}{2 \mu}\left(p_{0}^{2}+\frac{\hbar^{2}}{2 b^{2}}\right) .
$$

We will use the notation $\mu$ for the particle mass to avoid confusion with various quantum numbers.

If we expand such an initial state in terms of the energy eigenstates $u_{n}(x)$ of a general $1 \mathrm{D}$ bound state potential, we have

$$
\psi_{G}(x, 0)=\sum_{n=1}^{\infty} a_{n} u_{n}(x),
$$

with

$$
a_{n}=\int_{-\infty}^{+\infty} u_{n}^{*}(x) \psi_{G}(x, 0) d x
$$

The resulting $a_{n}$ must satisfy two constraints, namely,

$$
\begin{aligned}
\sum_{n=1}^{\infty}\left|a_{n}\right|^{2} & =1 \\
\sum_{n=1}^{\infty}\left|a_{n}\right|^{2} E_{n} & =\langle\hat{E}\rangle=\frac{1}{2 \mu}\left(p_{0}^{2}+\frac{\hbar^{2}}{2 b^{2}}\right),
\end{aligned}
$$

where $E_{n}$ represents the quantized energies of the potential. Equation (8) is very useful for numerical checks on the expansion coefficients. (We note that even if the subsequent time development of the quantum wave packet involves "collisions" with an infinite wall, if the wave packet is initially normalized to unit probability, it will remain so through its subsequent time development. Once we normalize the wave packet, unitarity ensures that we can "set it and forget it.") The resulting time-dependence is then given by

$$
\psi(x, t)=\sum_{n=1}^{\infty} a_{n} u_{n}(x) e^{-i E_{n} t / \hbar},
$$

and we know 11,20 that for short times (before the initially Gaussian packet has a chance to "collide" with any of the infinite wall boundaries), its behavior will resemble that of a free-particle solution of the form

$$
|\psi(x, t)|^{2}=\frac{1}{b_{t} \sqrt{\pi}} e^{-\left(x-x_{0}-p_{0} t / \mu\right)^{2} / b_{t}^{2}}
$$


where

$$
\begin{aligned}
b_{t} & \equiv \alpha \hbar \sqrt{1+\left(t / t_{0}\right)^{2}}, \\
\Delta x_{t} & =\Delta x_{0} \sqrt{1+\left(t / t_{0}\right)^{2}}
\end{aligned}
$$

with

$$
t_{0} \equiv \frac{\mu b^{2}}{\hbar}=\left(\frac{2 \mu}{\hbar^{2}}\right) \Delta x_{0}^{2}
$$

which defines the time scale for wave packet spreading.

\section{B. General time-dependence}

For the expansion of a general wave packet as in Eq. (9), we typically expand the energy eigenvalues about the central value of the quantum number $n_{0}$ (assumed to be an integer for simplicity) used in the construction of the wave packet, namely,

$$
E(n) \approx E\left(n_{0}\right)+E^{\prime}\left(n_{0}\right)\left(n-n_{0}\right)+\frac{1}{2} E^{\prime \prime}\left(n_{0}\right)\left(n-n_{0}\right)^{2}+\frac{1}{6} E^{\prime \prime \prime}\left(n_{0}\right)\left(n-n_{0}\right)^{3}+\cdots
$$

We can express the time-dependence of each quantum eigenstate as

$$
\begin{aligned}
e^{-i E_{n} t / \hbar}= & \exp \left(-i / \hbar\left[E\left(n_{0}\right) t+\left(n-n_{0}\right) E^{\prime}\left(n_{0}\right) t+\frac{1}{2}\left(n-n_{0}\right)^{2} E^{\prime \prime}\left(n_{0}\right) t+\ldots\right]\right) \\
= & \exp \left(-i \omega_{0} t-2 \pi i\left(n-n_{0}\right) t / T_{\mathrm{cl}}-2 \pi i\left(n-n_{0}\right)^{2} t / T_{\text {rev }}\right. \\
& \left.\quad-2 \pi i\left(n-n_{0}\right)^{3} t / T_{\text {super }}+\ldots\right)
\end{aligned}
$$

in terms of which the classical period, quantum mechanical revival, and superrevival times are given respectively by

$$
\begin{aligned}
T_{\mathrm{cl}} & =\frac{2 \pi \hbar}{\left|E^{\prime}\left(n_{0}\right)\right|} \\
T_{\mathrm{rev}} & =\frac{2 \pi \hbar}{\left|E^{\prime \prime}\left(n_{0}\right)\right| / 2} \\
T_{\text {super }} & =\frac{2 \pi \hbar}{\left|E^{\prime \prime \prime}\left(n_{0}\right)\right| / 6} .
\end{aligned}
$$

The first term of Eq. (14) is an common overall phase that gives the same (trivial) timedependence to each state, while the second term describes the short-term, classical periodicity of the bound state system because it gives $e^{-2 \pi i\left(n-n_{0}\right)}=1$ for each state after one classical period, $T_{\mathrm{cl}}$. (An alternative derivation of the form $T_{\mathrm{cl}}=2 \pi \hbar /\left|E^{\prime}\left(n_{0}\right)\right|$ is given in 
Appendix A using a WKB approach.) Bound state wave packets (with the exception of the harmonic oscillator potential for which the energy spectrum is exactly linear in $n$ ) will typically spread significantly after a number of classical periods, entering a so-called collapsed phase, only to reform later in the form of a quantum revival, in which the spreading reverses itself (completely in the case of the infinite well, partially in the case of a general potential well) and the wave packet relocalizes. The revival time, $T_{\mathrm{rev}}$, describes the (typically longer) time scale for that behavior.

A standard tool for analyzing the short-term, semi-classical periodicity, and the long-term revival structure is the autocorrelation function defined by ${ }^{33}$

$$
\begin{aligned}
A(t) & \equiv \int_{-\infty}^{+\infty} \psi^{*}(x, t) \psi(x, 0) d x \\
& =\int_{-\infty}^{+\infty} \phi^{*}(p, t) \phi(p, 0) d p \\
& =\sum_{n=1}^{\infty}\left|a_{n}\right|^{2} e^{i E_{n} t / \hbar}
\end{aligned}
$$

which measures the degree of overlap of the initial wave function with itself at later times (in both position- and momentum space).

\section{ONE-DIMENSIONAL INFINITE WELL}

For the 1D infinite well of width $a$, the energy eigenvalues and eigenfunctions are given by

$$
\begin{aligned}
E_{n} & =\frac{p_{n}^{2}}{2 \mu}=\frac{\hbar^{2} \pi^{2} n^{2}}{2 \mu a^{2}} \equiv n^{2} E_{0} \\
u_{n}(x) & =\sqrt{\frac{2}{a}} \sin \left(\frac{n \pi x}{a}\right) .
\end{aligned}
$$

The classical period obtained from Eq. (15) (using the identification $\mu v_{n} \equiv p_{n}=n \pi \hbar / a$ ) is given by

$$
\frac{d E_{n}}{d n}=\frac{\hbar^{2} \pi^{2} n}{\mu a^{2}}=\frac{\hbar \pi}{a}\left(\frac{p_{n}}{\mu}\right)=\frac{\hbar \pi v_{n}}{a}
$$

which implies that

$$
T_{\mathrm{cl}}=\frac{2 \pi \hbar}{|d E / d n|}=\frac{2 a}{v_{n}},
$$

as expected. The revival time is given as

$$
T_{\mathrm{rev}}=\frac{4 \mu a^{2}}{\hbar \pi}=\frac{2 \pi \hbar}{E_{0}},
$$


and the revivals are exact in this case because $e^{-i E_{n} T_{\mathrm{rev}} / \hbar}=e^{-2 \pi i n^{2}}=1$ for all values of $n$. Because of the quadratic $n$-dependence, there are no higher order time scales beyond the classical period, $T_{\mathrm{cl}}$, and the quantum revival time, $T_{\mathrm{rev}}$.

The expansion coefficients for a general Gaussian wave packet of the form in Eq. (2) are given by

$$
a_{n}=\int_{0}^{a}\left[u_{n}(x)\right]\left[\psi_{G}(x, 0)\right] d x
$$

where we can do the integrations numerically over the finite $(0, a)$ interval. We show in Fig. 1 the results of such a numerical computation, both for the normalization, $\sum_{n=1}^{\infty}\left|a_{n}\right|^{2}$, and the average energy, $\sum_{n=1}^{\infty} E_{n}\left|a_{n}\right|^{2}$, for zero momentum $\left(p_{0}=0\right)$ wave packets as we vary the initial position, $x_{0}$ : we use here (and elsewhere) the numerical values

$$
\hbar=2 \mu=a=1
$$

and show results for values of $b=1 /(2 \sqrt{10})(1 / \sqrt{10})$ corresponding to initial spatial widths of $\Delta x_{0}=0.05(0.10)$ as shown by the solid (dashed) curves; we used the 40 lowest-lying energy eigenstates in the expansion. As expected, as we move the initial wave packet near the edge (and beyond), the probability of being inside the well decreases uniformly, and the wider wave packet "senses" the wall first, as shown at the bottom of Fig. 1. Initial wave packets that are sufficiently far from either wall can therefore be reliably "supported" inside the well, despite the fact that they do not explicitly satisfy the boundary conditions at $x=0$ and $x=a$.

Perhaps more unexpectedly, the average energy of these wave packets grows rather dramatically (from the $p_{0}=0$ value of $\left.\langle\hat{E}\rangle=\left(\hbar^{2} / 2 b^{2}\right) / 2 \mu\right)$ as the packet nears the wall. This behavior is due to the expansion in Eq. (7) attempting to reproduce the sharply discontinuous jump enforced by the edge of the well at $x=a$, which requires the inclusion of many shorter wavelength and hence higher energy/momentum component states. In fact, for a sufficiently poorly behaved discontinuous function, the evaluation of the kinetic energy in such a way may be ill-defined, ${ }^{34}$ and increase without bound as the number of component states is increased.

To avoid all such difficulties, we will henceforth assume that the initial Gaussian is sufficiently contained within the billiard footprint so that we make an exponentially small error by neglecting any overlap with the region outside the $1 \mathrm{D}$ or $2 \mathrm{D}$ well, and may ignore any discontinuity at the wall. In practice, this condition only requires the wave packet to be a 
few times $\Delta x_{0}=b / \sqrt{2}$ away from an infinite wall boundary. We can then extend the integration region from the finite $(0, a)$ interval to the entire 1D space, giving the (exponentially good) approximation for the expansion coefficients

$$
\begin{aligned}
\tilde{a}_{n} & \equiv \int_{-\infty}^{+\infty}\left[u_{n}(x)\right]\left[\psi_{G}(x, 0)\right] d x \\
& =\left(\frac{1}{2 i}\right) \sqrt{\frac{4 b \pi}{a \sqrt{\pi}}}\left[e^{i n \pi x_{0} / a} e^{-b^{2}\left(p_{0}+n \pi \hbar / a\right)^{2} / 2 \hbar^{2}}-e^{-i n \pi x_{0} / a} e^{-b^{2}\left(p_{0}-n \pi \hbar / a\right)^{2} / 2 \hbar^{2}}\right],
\end{aligned}
$$

because we can write

$$
\sin \left(\frac{n \pi x}{a}\right)=\frac{1}{2 i}\left(e^{i n \pi x / a}-e^{-i n \pi x / a}\right),
$$

and we can perform Gaussian integrals such as

$$
\int_{-\infty}^{+\infty} e^{-a x^{2}-b x} d x=\sqrt{\frac{\pi}{a}} e^{b^{2} / 4 a}
$$

in closed form. This expression is very useful because it can speed up numerical calculation involving the expansion coefficients such as the evaluation of the autocorrelation function in Eq. (18). It also accurately encodes the sometimes delicate interplay between the oscillatory pieces of the Gaussian $\left(e^{-i p_{0} x / \hbar}\right)$ and the bound state $\left(e^{ \pm i n \pi x / a}\right)$ wavefunctions, which can be difficult to reproduce in a purely numerical evaluation, and it does so in a way that is valid for arbitrarily large values of $p_{0}$, where the integrand would be highly oscillatory. Finally, Eq. (26) nicely illustrates how $\psi_{G}(x, 0)$ and the $u_{n}(x)$ must not only have an appropriate overlap in position space, but also must have an appropriate phase relationship between their oscillatory terms. This phase connection leads to the $\exp \left(-b^{2}\left(p_{0} \pm n \pi \hbar / a\right)^{2} / 2 \hbar^{2}\right)$ terms, which can be understood from a complementary overlap in momentum space. (We discuss this point in more detail, as well as the evaluation of the $\tilde{a}_{n}$ in momentum space in Appendix B.)

We can make immediate use of Eq. (26) by considering zero momentum $\left(p_{0}=0\right)$ wave packets; this case corresponds to placing an object "at rest" inside the infinite well potential. For such cases, the only natural periodicity in the problem is the revival time in Eq. (23), because there is no classical periodic motion. In this special case, the expression for $\tilde{a}_{n}$ in Eq. (26) simplifies even further to

$$
\tilde{a}_{n}=\sqrt{\frac{4 b \pi}{a \sqrt{\pi}}} e^{-b^{2} n^{2} \pi^{2} / a^{2}} \sin \left(\frac{n \pi x_{0}}{a}\right),
$$


which shows that for several special values of $x_{0}$ in the well, several of the expansion coefficients will vanish for obvious symmetry reasons. For example, for $x_{0} / a=1 / 2$, all of the even $n=2,4,6, \ldots$ coefficients are zero and the only non-vanishing terms in the expansion are the odd ones $(n=2 k+1)$ which have energies of the form

$$
E_{k}=\frac{\hbar^{2} \pi^{2}}{2 \mu a^{2}}(2 k+1)^{2}=E_{0}\left(4 k^{2}+4 k+1\right)=E_{0}+8 E_{0}\left[\frac{k(k+1)}{2}\right] .
$$

The first term in Eq. (30) contributes only to the same overall phase to the time-dependence of each term. The second term is of the form $8 E_{0}$ times an integer and leads to revival times that are 8 times shorter than the standard $T_{\mathrm{rev}}=2 \pi \hbar / E_{0}$ in Eq. (23). Similarly, for the cases of $x_{0} / a=1 / 3,2 / 3$, the $a_{n}$ with $n=3 k$ vanish, leading to special exact revivals at multiples of $T_{\text {rev }} / 3$ for these two initial locations. (It is also possible to construct odd-parity wave packets that have different patterns of special revivals at other locations in the well.)

To illustrate both the revivals and fractional revivals in special cases, we plot in Fig. 2 the autocorrelation function, $|A(t)|^{2}$, for one revival time, $T_{\text {rev }}$, for different initial central positions, $x_{0} / a$, of the zero-momentum packet from the center to as near one edge as we can. We note that in each case there is an exact revival at $T_{\text {rev }}=2 \pi \hbar / E_{0}$, while for the special cases of $x_{0} / a=1 / 2$ and $2 / 3$, we find exact revivals at shorter time intervals as expected. For $x_{0} / a=0.8$, we also notice large partial (but not exact) revivals at $0.4 T_{\text {rev }}$ and $0.6 T_{\text {rev }}$ for similar reasons (because $\sin (4 n \pi / 5)$ vanishes for $n=5 k$.)

We next show in Fig. 3 the effect of "turning on" momentum values for an $x_{0}=a / 2$ wave packet. For $p_{0}=0$ (top line), we have the special pattern of exact revivals at multiples of $T_{\text {rev }} / 8$ noted above, due to the vanishing of the even expansion coefficients (shown in the corresponding $\left|a_{n}\right|^{2}$ versus $n$ plot in the right column). For a small, non-zero value ( $p_{0}=3 \pi$, second row), only the exact revival at $T_{\text {rev }}$ remains, because the even expansion coefficients are no longer forced to vanish. The autocorrelation function decreases somewhat more rapidly from its initial value than in the $p_{0}=0$ case, because the particle is slowly moving away from its initial position, in addition to spreading out.

For still larger values of momentum, such as $p_{0}=40 \pi$ (third row), we see obvious evidence for the classical periodicity and the first appearance of fractional revivals ${ }^{26}$ at rational fraction multiples of $T_{\text {rev }}$. The corresponding $a_{n}$ now exhibit a more obvious Gaussian shape, with a spread, $\Delta n$, which is related to the momentum variable by $p_{n}=n \pi \hbar / a$, so that

$$
\frac{\hbar}{2 \Delta x_{0}}=\frac{\hbar}{\sqrt{2} b}=\Delta p=\frac{\pi \hbar}{a} \Delta n
$$


which gives $\Delta n=a / 2 \pi \Delta x_{0}$. (This relation can be useful in deciding how many values of $a_{n}$ to include in a numerical evaluation of quantities such as $A(t)$.) For even larger momentum values (see the $p_{0}=400 \pi$ case at the bottom, for example), the classical period becomes much shorter than any obvious fractional revival time scale, and the shape of the expansion coefficient distribution is unchanged (same $\Delta n$ ), but simply shifted to higher values of $n$.

These results, especially the expression for the expansion coefficients in Eq. (26), can be used in the square billiard we consider in Sec. IV because that geometry is clearly separable into two 1D problems.

\section{SQUARE BILLIARD}

\section{A. Time-dependence for two-dimensional systems}

Systems with two quantum numbers ${ }^{35,36}$ with energy $E\left(n_{1}, n_{2}\right)$ offer richer possibilities for both semi-classical periodicities as well as wave packet revivals. The extension of Eq. (13) gives two possible classical periods, namely

$$
\begin{aligned}
T_{\mathrm{cl}}^{\left(n_{1}\right)} & =\frac{2 \pi \hbar}{\left|\partial E\left(n_{1}, n_{2}\right) / \partial n_{1}\right|} \\
T_{\mathrm{cl}}^{\left(n_{2}\right)} & =\frac{2 \pi \hbar}{\left|\partial E\left(n_{1}, n_{2}\right) / \partial n_{2}\right|},
\end{aligned}
$$

and the long-time revival structure typically depends on three possible times given by

$$
\begin{aligned}
T_{\mathrm{rev}}^{\left(n_{1}\right)} & =\frac{2 \pi \hbar}{(1 / 2)\left|\partial^{2} E\left(n_{1}, n_{2}\right) / \partial n_{1}^{2}\right|} \\
T_{\mathrm{rev}}^{\left(n_{2}\right)} & =\frac{2 \pi \hbar}{(1 / 2)\left|\partial^{2} E\left(n_{1}, n_{2}\right) / \partial n_{2}^{2}\right|} \\
T_{\mathrm{rev}}^{\left(n_{1}, n_{2}\right)} & =\frac{2 \pi \hbar}{\left|\partial^{2} E\left(n_{1}, n_{2}\right) / \partial n_{1} \partial n_{2}\right|},
\end{aligned}
$$

and the revival structure depends on the interplay between these three times.

Classical closed or periodic orbits, with periods given by $T_{\mathrm{cl}}^{(\mathrm{po})}$, are reproduced when the two classical periods are commensurate, namely, when

$$
p T_{\mathrm{cl}}^{\left(n_{1}\right)}=T_{\mathrm{cl}}^{(\mathrm{po})}=q T_{\mathrm{cl}}^{\left(n_{2}\right)}
$$

The relation (34) can be interpreted as arising from the beating of the two classical periods against each other ${ }^{35}$ 


\section{B. Time-dependence for the square billiard}

For the infinite square well (with dimensions $L_{x} \times L_{y}=a \times a$ ), the problem simplifies to two copies of a single 1D infinite well because of the separability of the potential. For example, the energy eigenvalues, $E\left(n_{x}, n_{y}\right)$, and eigenstates, $w_{\left(n_{x}, n_{y}\right)}(x, y)$, are given by

$$
E\left(n_{x}, n_{y}\right)=\frac{\hbar^{2} \pi^{2}\left(n_{x}^{2}+n_{y}^{2}\right)}{2 \mu a^{2}}
$$

and

$$
w_{\left(n_{x}, n_{y}\right)}(x, y)=u_{\left(n_{x}\right)}(x) u_{\left(n_{y}\right)}(y),
$$

where $n_{x}, n_{y}=1,2,3, \ldots$ are the appropriate quantum numbers. The revival times are given by Eqs. (33a) and (33b), and are simply related to each other via

$$
T_{\mathrm{rev}}^{\left(n_{x}\right)}=\frac{4 \mu a^{2}}{\hbar \pi}=T_{\mathrm{rev}}^{\left(n_{y}\right)}
$$

with no cross-term present. Therefore, the quantum revival structure also is very simply related to that of the $1 \mathrm{D}$ infinite well, including the possibilities of special symmetric revivals for zero-momentum wave packets at particular locations, such as $\left(x_{0}, y_{0}\right)=(a / 2, a / 2)$ and $(a / 3,2 a / 3)$. For rectangular infinite wells with incommensurate sides $\left(L_{x} / L_{y} \neq p / q\right)$, the structure of the revival times may be more complex. ${ }^{35,36}$

Compared to the simple back-and-forth motions in the 1D infinite well, the closed or periodic orbits here are more interesting and the nontrivial path lengths for closed orbits, $L(p, q)$, in the square billiard can be readily deduced from simple geometric arguments, $, 37,38$ and are given by

$$
L(p, q)=2 a \sqrt{p^{2}+q^{2}}
$$

where $2 p$ and $2 q$ count the number of "hits" on the horizontal and vertical walls respectively, before returning to the starting point in phase space. The corresponding classical periods for such closed trajectories would be given by

$$
T_{\mathrm{cl}}^{(\mathrm{po})}=\frac{L(p, q)}{v_{0}},
$$

where $v_{0}$ is the classical speed. Such orbits can be produced by point particles in the 2D billiard, starting from any initial location, $\left(x_{0}, y_{0}\right)$, inside the box, provided they are pointed appropriately, namely in the $\tan (\theta)=q / p$ direction. The values of $\theta=\tan ^{-1}(q / p)$ and 
$T_{\mathrm{cl}}^{(\mathrm{po})} / \tau=\sqrt{p^{2}+q^{2}}$ (where $\tau \equiv 2 a / v_{0}$ is the period for the simplest, back-and-forth closed trajectory) for many of the low-lying cases are tabulated in Table I.

The condition for periodic orbits in Eq. (34) can be implemented very easily in this case, and we will examine its derivation in detail. For such closed orbits to occur we require that

$$
p\left(\frac{2 \mu a^{2}}{\hbar \pi n_{x}}\right)=p T_{\mathrm{cl}}^{\left(n_{x}\right)}=q T_{\mathrm{cl}}^{\left(n_{y}\right)}=q\left(\frac{2 \mu a^{2}}{\hbar \pi n_{y}}\right),
$$

or $n_{y}=n_{x}(q / p)$. If we substitute Eq. (39) into Eq. (35), as well as equate the quantized total energy, $E\left(n_{x}, n_{y}\right)$, with the classical kinetic energy, we obtain

$$
\begin{aligned}
\frac{1}{2} \mu v_{0}^{2} \longleftrightarrow E\left(n_{x}, n_{y}\right) & =\frac{\hbar^{2} \pi^{2}}{2 \mu a^{2}}\left(n_{x}^{2}+n_{y}^{2}\right) \\
& =\frac{\hbar^{2} \pi^{2}}{2 \mu a^{2}}\left[n_{x}^{2}+n_{x}^{2}\left(\frac{q}{p}\right)^{2}\right] \\
& =\frac{\hbar^{2} \pi^{2}}{2 \mu a^{2}}\left[\frac{n_{x}^{2}\left(p^{2}+q^{2}\right)}{p^{2}}\right],
\end{aligned}
$$

or

$$
n_{x}=\frac{\mu a v_{0}}{\hbar \pi} \frac{p}{\sqrt{p^{2}+q^{2}}} \quad \text { and } \quad n_{y}=\frac{\mu a v_{0}}{\hbar \pi} \frac{q}{\sqrt{p^{2}+q^{2}}}
$$

so that

$$
T_{\mathrm{cl}}^{(\mathrm{po})}=p T_{\mathrm{cl}}^{\left(n_{x}\right)}=p\left(\frac{2 \mu a^{2}}{\hbar \pi n_{x}}\right)=\frac{2 a \sqrt{p^{2}+q^{2}}}{v_{0}} .
$$

Equation (42) is consistent with the purely classical and geometrical result from Eq. (38).

We can visualize the appearance of these closed orbits in the time development of the quantum solutions of the Schrödinger equation by constructing 2D Gaussian wave packets of the form

$$
\psi_{G}(x, y ; t=0)=\psi_{G}\left(x ; x_{0}, p_{0 x}, b\right) \psi_{G}\left(y ; y_{0}, p_{0 y}, b\right)
$$

where

$$
\psi_{G}\left(x ; x_{0}, p_{0 x}, b\right)=\frac{1}{\sqrt{b \sqrt{\pi}}} e^{-\left(x-x_{0}\right)^{2} / 2 b^{2}} e^{i p_{0 x}\left(x-x_{0}\right) / \hbar} .
$$

A similar expression holds for $\psi_{G}\left(y ; y_{0}, p_{0 y}, b\right)$. Once again, as long as the initial location, $\left(x_{0}, y_{0}\right)$, is far away from the edges of the potential well, such a Gaussian form can be easily expanded in terms of the eigenstates. The various position and momentum space expectation values are given by analogs of Eqs. (41) and (5), and the expectation value of total energy is now

$$
\langle\hat{E}\rangle=\frac{1}{2 \mu}\left\langle\hat{p}_{x}^{2}+\hat{p}_{y}^{2}\right\rangle=\frac{1}{2 \mu}\left[p_{0 x}^{2}+p_{0 y}^{2}+\frac{\hbar^{2}}{b^{2}}\right] .
$$


The expansion coefficients also exhibit separability with

$$
\begin{aligned}
\psi_{G}(x, y ; t=0) & =\sum_{n_{x}=1}^{\infty} \sum_{n_{y}=1}^{\infty} a_{n_{x}} a_{n_{y}} u_{\left(n_{x}\right)}(x) u_{\left(n_{y}\right)}(y) e^{-i E\left(n_{x}, n_{y}\right) t / \hbar} \\
& =\left[\sum_{n_{x}=1}^{\infty} a_{n_{x}} u_{\left(n_{x}\right)}(x) e^{-i E\left(n_{x}\right) t / \hbar}\right]\left[\sum_{n_{y}=1}^{\infty} a_{n_{y}} u_{\left(n_{y}\right)}(y) e^{-i E\left(n_{y}\right) t / \hbar}\right] \\
& =\psi_{G}(x, t) \psi_{G}(y, t) .
\end{aligned}
$$

with an equally simple expression for the autocorrelation function, namely

$$
A(t)=A_{x}(t) A_{y}(t)
$$

We illustrate the time-dependence of such wave packets by plotting $|A(t)|^{2}=$ $\left|A_{x}(t) A_{y}(t)\right|^{2}$ versus $t$ in Fig. 4. The results shown are for wave packets characterized by initial positions $\left(x_{0}, y_{0}\right)=(a / 2, a / 2)$ and initial momenta given by $\left(p_{0 x}, p_{0 y}\right)=$ $\left(p_{0} \cos (\theta), p_{0} \sin (\theta)\right)$ where $p_{0}=400 \pi$ and $\theta=\tan ^{-1}\left(p_{0 y} / p_{0 x}\right) ;$ we also use $\Delta x_{0}=\Delta y_{0}=0.05$ and the same physical parameters as in Eq. (25). With these values, the classical period (for the simplest back-and-forth motion), $T_{\mathrm{cl}}=2 a / v_{0}$, the spreading time, $t_{0}$ (from Eq. (12)), and the revival time, $T_{\text {rev }}$ (from Eq. (36) ), are given by $T_{\mathrm{cl}}=2 \mu a / p_{0}=1 /(400 \pi) \approx 0.8 \times 10^{-3}$, $t_{0}=(2 \mu / \hbar) \Delta x_{0}^{2}=(0.05)^{2}=2.5 \times 10^{-3}$, and $T_{\mathrm{rev}}=4 \mu a^{2} /(\hbar \pi)=2 / \pi \approx 0.64$. The wave packet will then exhibit a reasonable number of classical periods before significant spreading occurs, with the revival time scale being much larger than both (which is a typical hierarchy for the time scales for physical revivals, such as seen in Rydberg atom states. ${ }^{1}$ )

We show $|A(t)|^{2}$ versus $t$ in Fig. 4 for increasing values of $\theta$ up to $45^{\circ}$ (about which the pattern of classical orbits and autocorrelation function data are symmetric). We indicate the expected location of the classical closed or periodic orbits by stars at locations of values of $T_{\mathrm{cl}}^{(\mathrm{po})} / \tau=\sqrt{p^{2}+q^{2}}$ and $\theta=\tan ^{-1}(q / p)$ corresponding to the values in Table I. (Images of some of the simplest classical closed orbits are also included at the far right for the appropriate values of $\theta$.) We note that the classical periodicities for closed orbits are obviously reproduced in the time-development of the appropriate quantum wave packets. We have also confirmed that varying the initial location of the wave packet has no affect on the results shown in Fig. 4, consistent with the classical result that the existence of closed orbits depend only on $p, q$ through $p_{0 y} / p_{0 x}=\tan (\theta)=q / p$. 


\section{Special triangular billiards}

The energy eigenvalues and wavefunctions for a special 2D triangular billiard can be easily derived from those of the infinite square well solutions in Eq. (35)). The standard results for the square well, $E\left(n_{x}, n_{y}\right)=\left(\hbar^{2} \pi^{2} / 2 \mu a^{2}\right)\left(n_{x}^{2}+n_{y}^{2}\right)$ and $w_{\left(n_{x}, n_{y}\right)}(x, y)=u_{\left(n_{x}\right)}(x) u_{\left(n_{y}\right)}(y)$, hold for any integral $n_{x}, n_{y} \geq 1$; for $n_{x}=n_{y}$ there is a single state, while for $n_{x} \neq n_{y}$, there is a two-fold degeneracy. Linear combinations of these solutions can be written in the form

$$
\begin{aligned}
w_{(n, m)}^{(-)}(x, y)=\frac{1}{\sqrt{2}}\left[u_{(n)}(x) u_{(m)}(y)-u_{(m)}(x) u_{(n)}(y)\right] & (m \neq n) \\
w_{(n, m)}^{(+)}(x, y)=\frac{1}{\sqrt{2}}\left[u_{(n)}(x) u_{(m)}(y)+u_{(m)}(x) u_{(n)}(y)\right] & (m \neq n) \\
w_{(n, n)}^{(0)}(x, y)=u_{(n)}(x) u_{(n)}(y), &
\end{aligned}
$$

which have the same energy degeneracy, but exhibit different patterns of nodal lines. These alternative forms are useful because they allow one to discuss the energy eigenvalues and eigenfunctions of the $45^{\circ}-45^{\circ}-90^{\circ}$ triangle billiard ${ }^{39.40 .41}$ formed by "folding" the square along

a diagonal, because the $w_{(n, m)}^{(-)}(x, y)$ satisfy the appropriate boundary condition along the new hypotenuse. (We can easily see from Eq. (49a) that $w_{(n, m)}^{(-)}(x, y=x)=0$ by construction.) Additional foldings along diagonals are also possible, $\frac{41}{\underline{1}}$ and the energy spectrum can be used to analyze these cases as well. The allowed eigenvalues for this case are still given by $E\left(n_{x}, n_{y}\right)=\left(\hbar^{2} \pi^{2} / 2 \mu a^{2}\right)\left(n_{x}^{2}+n_{y}^{2}\right)$, which corresponds to

$$
E(n, m)=\frac{\hbar^{2} \pi^{2}}{2 \mu a^{2}}\left(n^{2}+m^{2}\right),
$$

but now with only a single $\left(n_{x}, n_{y}\right) \rightarrow(n, m)$ state allowed, with corresponding wavefunctions given by Eq. (49a), but multiplied by $\sqrt{2}$ to account for the different normalization needed in the smaller area billiard.

The revival time in the $45^{\circ}-45^{\circ}-90^{\circ}$ triangular billiard is still given by Eq. (36) and localized wave packets can also be constructed using the appropriately normalized analogs of the wavefunctions in Eq. (49), again provided they are kept away from any of the infinite wall boundaries.

The structure of the classical closed or periodic orbits in this case is the same as for the square billiard, because all of the standard $(p, q)$ orbits in the square well are simply reflected off the new diagonal wall (along the hypotenuse), giving rise to the same allowed orbits as in Eq. (37) and Table I. The only new feature in the semi-classical propagation of such 
wave packet solutions in this folded geometry ${ }^{41}$ is the existence of a special isolated closed orbit at $135^{\circ}$ (one that bisects the $90^{\circ}$ right angle, bouncing normally off the hypotenuse, and returning to the corner), which has path lengths that are multiples of $\left(\sqrt{1^{2}+1^{2}}\right) a / 2=$ $\sqrt{2} a / 2$, namely half that of the standard $(p, q)=(1,1)$ features. When we construct wave packets using parameters appropriate to this geometry, we see twice as many features in the $A(t)$ plot for this case because of this special classical closed orbit, but we otherwise reproduce the results shown in Fig. 4.

\section{EQUILATERAL TRIANGLE BILLIARDS}

It is perhaps under appreciated that the energy eigenvalues and position space wave functions for a particle of mass $\mu$ in an equilateral triangular $\left(60^{\circ}-60^{\circ}-60^{\circ}\right)$ infinite well (or billiard) of side $a$ are available in closed form. They have been derived for a variety of different contexts by at least four groups ${ }^{42.43 .44 .45}$ using complementary methods of derivation.

For definiteness in what follows, we will assume such a triangular billiard with vertices located at $(0,0),(a / 2, \sqrt{3} a / 2)$, and $(-a / 2, \sqrt{3} a / 2)$. The resulting energy spectrum is given by

$$
E(m, n)=\frac{\hbar^{2}}{2 \mu a^{2}}\left(\frac{4 \pi}{3}\right)^{2}\left(m^{2}+n^{2}-m n\right)
$$

for integral values of $m$ and $n$, with the restriction that $m \geq 2 n$. (In what follows, we will use the notation of Ref. 43, except for a minor relabeling of the energies and wavefunctions: we will continue to use $\mu$ for the particle mass to avoid confusion with various quantum

numbers.) For $m>2 n$, there are two degenerate states with different symmetry properties ${ }^{43}$ that can be written in the forms

$$
\begin{array}{r}
w_{(m, n)}^{(-)}(x, y)=\sqrt{\frac{16}{a^{2} 3 \sqrt{3}}}\left[\sin \left(\frac{2 \pi(2 m-n) x}{3 a}\right) \sin \left(\frac{2 \pi n y}{\sqrt{3} a}\right)\right. \\
-\sin \left(\frac{2 \pi(2 n-m) x}{3 a}\right) \sin \left(\frac{2 \pi m y}{\sqrt{3} a}\right) \\
\left.-\sin \left(\frac{2 \pi(m+n) x}{3 a}\right) \sin \left(\frac{2 \pi(m-n) y}{\sqrt{3} a}\right)\right],
\end{array}
$$


and

$$
\begin{array}{r}
w_{(m, n)}^{(+)}(x, y)=\sqrt{\frac{16}{a^{2} 3 \sqrt{3}}}\left[\cos \left(\frac{2 \pi(2 m-n) x}{3 a}\right) \sin \left(\frac{2 \pi n y}{\sqrt{3} a}\right)\right. \\
-\cos \left(\frac{2 \pi(2 n-m) x}{3 a}\right) \sin \left(\frac{2 \pi m y}{\sqrt{3} a}\right) \\
\left.+\cos \left(\frac{2 \pi(m+n) x}{3 a}\right) \sin \left(\frac{2 \pi(m-n) y}{\sqrt{3} a}\right)\right] .
\end{array}
$$

We can confirm by direct differentiation that they satisfy the 2D Schrödinger equation, with the energy eigenvalues in Eq. (51), as well as the appropriate boundary conditions. We have here also included the correct normalizations, because we will eventually expand Gaussian wave packets in these eigenstates.

For the special case of $m=2 n$, there is a single non-degenerate state for each value of $n$ given by

$$
w_{(2 n, n)}^{(0)}(x, y)=\sqrt{\frac{8}{a^{2} 3 \sqrt{3}}}\left[2 \cos \left(\frac{2 \pi n x}{a}\right) \sin \left(\frac{2 \pi n y}{\sqrt{3} a}\right)-\sin \left(\frac{4 \pi n y}{\sqrt{3} a}\right)\right] .
$$

Clearly these states satisfy

$$
\begin{aligned}
& w_{(m, n)}^{( \pm)}(-x, y)= \pm w_{(m, n)}^{( \pm)}(x, y) \\
& w_{(m, n)}^{(0)}(-x, y)=+w_{(m, n)}^{(0)}(x, y) .
\end{aligned}
$$

and the $w_{(m=2 n, n)}^{( \pm)}(x, y)$ states also satisfy

$$
\begin{aligned}
& w_{(m=2 n, n)}^{(+)}(x, y)=\sqrt{2} w_{(2 n, n)}^{(0)}(x, y) \\
& w_{(m=2 n, n)}^{(-)}(x, y)=0 .
\end{aligned}
$$

The pattern of energy level degeneracies and wavefunction symmetries is thus very similar to that for the square billiard, especially when the solutions for that problem are written in the form of Eq. (49).

We note that the corresponding momentum space wavefunctions, $f_{(m, n)}^{( \pm)}\left(p_{x}, p_{y}\right)$, can also be obtained in closed form, because the required 2D Fourier transform over the triangular region can be done using standard integrals involving trigonometric functions and powers, making this problem very useful for still another reason.

We turn now to the time-dependence of wave packets in this geometry and consider the 
long-term, revival time scales in this two quantum number system:

$$
\begin{aligned}
T_{\mathrm{rev}}^{(m)} & =\frac{2 \pi \hbar}{\left|\partial^{2} E / \partial m^{2}\right| / 2} \\
T_{\mathrm{rev}}^{(n)} & =\frac{2 \pi \hbar}{\left|\partial^{2} E / \partial n^{2}\right| / 2} \\
T_{\mathrm{rev}}^{(m, n)} & =\frac{2 \pi \hbar}{\left|\partial^{2} E / \partial m \partial n\right|},
\end{aligned}
$$

which gives

$$
T_{\mathrm{rev}}^{(m)}=T_{\mathrm{rev}}^{(n)}=T_{\mathrm{rev}}^{(m, n)} \equiv T_{\mathrm{rev}}=\frac{9 \mu a^{2}}{4 \hbar \pi} .
$$

Exact revivals are present in this system (just as for the square well), with a single revival time guaranteed for any possible initial wave packets. Thus, both the $N=3$ and $N=4$ polygonal billiards (the equilateral triangle and square) exhibit similar and simple energy eigenvalues and exact quantum revivals. We note that special, shorter-time scale revivals are also possible in the equilateral triangle case for zero-momentum wave packets initially centered at symmetric locations within the triangular billiard, ${ }^{32}$ such as at the geometrical center and half-way down a bisector.

The short-time classical periodicity of quantum wave packets in this geometry can also be determined from calculations of

$$
\begin{aligned}
& T_{\mathrm{cl}}^{(m)}=\frac{2 \pi \hbar}{|\partial E / \partial m|}=\left[\frac{9 \mu a^{2}}{4 \hbar \pi}\right] \frac{1}{(2 m-n)} \\
& T_{\mathrm{cl}}^{(n)}=\frac{2 \pi \hbar}{|\partial E / \partial n|}=\left[\frac{9 \mu a^{2}}{4 \hbar \pi}\right] \frac{1}{(2 n-m)} .
\end{aligned}
$$

The condition leading to closed orbits can then be written as

or

$$
\frac{(2 m-n)}{(2 n-m)}=\frac{T_{\mathrm{cl}}^{(n)}}{T_{\mathrm{cl}}^{(m)}}=\frac{p}{q}
$$

$$
n=m\left(\frac{2 p+q}{2 q+p}\right)
$$

If we substitute this condition into the energy spectrum in Eq. (51) and equate the quantum energies with the classical kinetic energy, $\mu v_{0}^{2} / 2$, we are led to the association of $\mu v_{0}^{2} / 2$ with $E(m, n)$ and

$$
E(m, n)=\left(\frac{16 \pi^{2}}{9}\right)\left(\frac{\hbar^{2}}{2 \mu a^{2}}\right)\left[m^{2}+m^{2}\left(\frac{2 p+q}{2 q+p}\right)^{2}-m^{2}\left(\frac{2 p+q}{2 q+p}\right)\right]
$$


or

$$
\left(\frac{2 \mu v_{0} a}{4 \pi \hbar}\right)^{2}=m^{2}\left[\frac{3\left(p^{2}+p q+q^{2}\right)}{(2 q+p)^{2}}\right] .
$$

This identification implies that

$$
\begin{aligned}
& m=\left(\frac{2 \mu v_{0} a}{4 \pi \hbar}\right) \frac{(2 q+p)}{\sqrt{3} \sqrt{p^{2}+p q+q^{2}}} \\
& n=\left(\frac{2 \mu v_{0} a}{4 \pi \hbar}\right) \frac{(2 p+q)}{\sqrt{3} \sqrt{p^{2}+p q+q^{2}}} .
\end{aligned}
$$

The period for classical, closed/periodic orbits is then given by

$$
T_{\mathrm{cl}}^{(\mathrm{po})}=p T_{\mathrm{cl}}^{(m)}=\frac{a \sqrt{3} \sqrt{p^{2}+p q+q^{2}}}{v_{0}}=\frac{L(p, q)}{v_{0}},
$$

where

$$
L(p, q)=d(p, q)=a \sqrt{3} \sqrt{p^{2}+p q+q^{2}}
$$

are the corresponding path lengths for periodic orbits. The possible classical closed or periodic orbits can be derived ${ }^{32}$ from a geometrical construction (involving tiling of the 2D plane, just as for the square $\operatorname{case}^{37.38}$ ) giving the same result. We note that the special cases of $2 m=n$ and $2 n=m$ correspond to $q=0$ and $p=0$ respectively, both of which give $L(p, q)=\sqrt{3} a$. In these cases, one of the classical periods from Eq. (59) formally goes to infinity, which can be understood classically from the corresponding path length, which corresponds to periodic back and forth motion from one corner, along a bisector, to the opposite side, but with no repetition in the complementary direction. (See Ref. 43 for a derivation of the quantized energies from which this effect also can be inferred.)

Because of the (relatively) simple form of the allowed wavefunctions in Eqs. (152) and (53), we can evaluate the expansion coefficients for any 2D Gaussian wave packet of the form in Eq. (43) by extending the region of integration from the (finite) triangular region to the entire $2 \mathrm{D}$ space, as long as the initial wave packet is far away from any of the walls. ${ }^{32}$ The required Gaussian-type integrals have the forms

$$
\begin{array}{r}
\int_{-\infty}^{+\infty} e^{i p_{0}\left(x-x_{0}\right) / \hbar} e^{-\left(x-x_{0}\right)^{2} / 2 b^{2}} \cos \left(\frac{C x}{a}\right) d x=\frac{b \sqrt{2 \pi}}{2}\left[e^{i C x_{0} / a} e^{-b^{2}\left(C / a+p_{0} / \hbar\right)^{2} / 2}\right. \\
\left.+e^{-i C x_{0} / a} e^{-b^{2}\left(-C / a+p_{0} / \hbar\right)^{2} / 2}\right]
\end{array}
$$

and

$$
\begin{array}{r}
\int_{-\infty}^{+\infty} e^{i p_{0}\left(x-x_{0}\right) / \hbar} e^{-\left(x-x_{0}\right)^{2} / 2 b^{2}} \sin \left(\frac{C x}{a}\right) d x=\frac{b \sqrt{2 \pi}}{2 i}\left[e^{i C x_{0} / a} e^{-b^{2}\left(C / a+p_{0} / \hbar\right)^{2} / 2}\right. \\
\left.-e^{-i C x_{0} / a} e^{-b^{2}\left(-C / a+p_{0} / \hbar\right)^{2} / 2}\right]
\end{array}
$$


with similar expressions for the $y$-integrations. The resulting closed form expressions for the expansion coefficients can be used in calculations of the autocorrelation function to illustrate the long-time revival structure of the wave packets, as well as the short-time, semi-classical propagation giving rise to closed orbits of the form in Eq. (64); the analogs of Figs. 2 and 4 for the equilateral triangle billiard have been presented in Ref. 32 .

A folding of the equilateral $\left(60^{\circ}-60^{\circ}-60^{\circ}\right)$ triangle along a bisector yields another special triangular geometry, namely a $30^{\circ}-60^{\circ}-90^{\circ}$ right triangle. The energy eigenvalues and eigenfunctions for this case can also be trivially obtained from those of Eq. (52) as they satisfy the new boundary condition along the fold. Such a system will have the same energy eigenvalues as in Eq. (51) (but with only one possible $(m, n)$ combination with $m>2 n$ ) and the same common revival time, $T_{\text {rev }}$, as in Eq. (58). Wave packet solutions can also be constructed (remembering to include an additional factor of $\sqrt{2}$ to account for the normalization difference) from the $w_{(m, n)}^{(-)}(x, y)$ in Eq. (152).

\section{CIRCULAR BILLIARDS}

\section{A. Eigenstate solutions and connections to classical closed orbits}

We finally turn our attention to the $N \rightarrow \infty$ limit of the $N$-sided regular polygonal billiard, namely the circular infinite well. (We note that the problem of scattering from an infinite cylindrical barrier in two dimensions as been considered in Ref. 46.) The potential for this problem can be defined by

$$
V_{C}(r)=\left\{\begin{array}{l}
0 \quad \text { for } r<R \\
\infty \text { for } r \geq R
\end{array} .\right.
$$

The (unnormalized) solutions of the corresponding 2D Schrödinger equation are given by

$$
u_{(m)}(r, \theta)=J_{|m|}(k r) e^{i m \theta}
$$

where the quantized angular momentum values are given by $L_{z}=m \hbar$ for $m=0, \pm 1, \pm 2, \ldots$. and the $J_{|m|}(k r)$ are the (regular) Bessel functions of order $|m|$.

The wavenumber, $k$, is related to the energy via $k=\sqrt{2 \mu E / \hbar^{2}}$ and the energy eigenvalues are quantized by the boundary conditions at the infinite wall at $r=R$, namely $J_{|m|}(z=$ 
$k R)=0$. The quantized energies are then given by

$$
E_{\left(m, n_{r}\right)}=\frac{\hbar^{2}\left[z_{\left(m, n_{r}\right)}\right]^{2}}{2 \mu R^{2}}
$$

where $z_{\left(m, n_{r}\right)}$ denotes the zeros of the Bessel function of order $|m|$, and $n_{r}$ counts the number of radial nodes.

The energy spectrum is doubly degenerate for $|m| \neq 0$ corresponding to the equivalence of clockwise and counter-clockwise $(m>0$ and $m<0)$ motion. We therefore see a pattern of degeneracies very similar to that of both the square and equilateral triangle wells, with two equal energy states for each $\left(|m|>0, n_{r}\right)$ value, and a single one for each $\left(m=0, n_{r}\right)$. Because the quantum number dependence of the energy eigenvalues is the determining factor in the structure of wave packet revivals, we need to examine the $m, n_{r}$ dependence of $E_{\left(m, n_{r}\right)} \propto\left[z_{\left(m, n_{r}\right)}\right]^{2}$.

As a first approximation, we can look at the large $z$ behavior of the Bessel function solutions $\frac{47}{}$ for fixed values of $|m|$, namely

$$
J_{|m|}(z) \longrightarrow \sqrt{\frac{2}{\pi z}} \cos \left(z-\frac{|m|}{2}-\frac{\pi}{4}\right)+\cdots
$$

With this approximation, the zeros would be given by $z_{\left(m, n_{r}\right)}-\frac{|m|}{2}-\frac{\pi}{4} \approx\left(2 n_{r}+1\right) \frac{\pi}{2}$, or

$$
z_{\left(m, n_{r}\right)} \approx\left(n_{r}+\frac{|m|}{2}+\frac{3}{4}\right) \pi
$$

If this result were exact, the quantized energies would depend quadratically on two quantum numbers, and there would be exact wave packet revivals, just as for the square or equilateral triangle billiards. However, there are important corrections to this first-order result, which means that the Bessel function zeros are not given by exact integral values. So although there are not exact quantum revivals in the general case, approximate revivals are present, most especially for zero-momentum, central $\left(\left(x_{0}, y_{0}\right)=(0,0)\right)$ wave packets (constructed from purely $m=0$ states). We refer the interested reader to Ref. 31 for details.

We can, however, examine the short-time dependence leading to classical closed orbits by making use of the WKB approximation to evaluate the quantized energies and their $m$ and $n_{r}$ dependence. If we first quantize the angular variable to find that the angular momentum is given by $L_{z}=m \hbar$, we note that in the radial direction the particle moves freely up to the infinite wall at $r=R$, but is subject to an effective centrifugal potential given by $V_{\text {eff }}(r)=L_{z}^{2} / 2 \mu r^{2}=(m \hbar)^{2} / 2 \mu r^{2}$. A classical particle cannot penetrate this centrifugal 
barrier and therefore has an inner radius (distance of closest approach) given by $V_{\text {eff }}\left(R_{\min }\right)=$ $m^{2} \hbar^{2} /\left(2 \mu R_{\text {min }}^{2}\right)=E$, or

$$
R_{\min }=\frac{|m| \hbar}{\sqrt{2 \mu E}} .
$$

We can also write Eq. (173) in the useful form $R_{\min }=|m| R / z$ and $E \equiv \hbar^{2} z^{2} /\left(2 \mu R^{2}\right)$ in terms of the dimensionless variable, $z$.

The WKB quantization condition on the radial variable $r$ is then given by

where

$$
\int_{R_{\min }}^{R} k_{r}(r) d r=\left(n_{r}+C_{L}+C_{R}\right) \pi
$$

$$
k_{r}(r) \equiv \sqrt{\frac{2 \mu E}{\hbar^{2}}} \sqrt{1-\frac{R_{\min }^{2}}{r^{2}}} .
$$

The matching coefficients $\frac{48}{*}$ are given by $C_{L}=1 / 4$ and $C_{R}=1 / 2$ which are appropriate for linear boundaries (at the inner centrifugal barrier) and hard or infinite wall boundaries (such as at $r=R$ ), respectively. (See Appendix A for more discussion of WKB methods.) The WKB energy quantization condition for the quantized energies in terms of $n_{r}$ explicitly and $|m|$ implicitly can then be written in the form

$$
\sqrt{\frac{2 \mu}{\hbar^{2}}} \int_{R_{\min }}^{R} \sqrt{E-\frac{m^{2} \hbar^{2}}{2 \mu r^{2}}} d r=\sqrt{\frac{2 \mu E}{\hbar^{2}}} \int_{R_{\min }}^{R} \sqrt{1-\frac{R_{\min }^{2}}{r^{2}}} d r=\left(n_{r}+3 / 4\right) \pi,
$$

which defines the quantized energies, $E=E_{\left(m, n_{r}\right)}$, implicitly in terms of $m, n_{r}$. To obtain the partial derivatives that are necessary to evaluate the classical periods in Eq. (32), we can then differentiate this condition with respect to both quantum numbers to obtain

$$
\begin{aligned}
\sqrt{\frac{\mu}{2 \hbar^{2}}}\left[\int_{R_{\min }}^{R} \frac{d r}{\sqrt{E-m^{2} \hbar^{2} / 2 \mu r^{2}}}\right]\left(\frac{\partial E}{\partial n_{r}}\right) & =\pi \\
\sqrt{\frac{\mu}{2 \hbar^{2}}}\left[\int_{R_{\min }}^{R} \frac{d r}{\sqrt{E-m^{2} \hbar^{2} / 2 \mu r^{2}}}\left(\frac{\partial E}{\partial m}-\frac{|m| \hbar^{2}}{\mu r^{2}}\right)\right] & =0 .
\end{aligned}
$$

The condition for periodic orbits from Eq. (34) can then be written as

$$
\frac{q}{p}=\frac{T_{\mathrm{cl}}^{\left(n_{r}\right)}}{T_{\mathrm{cl}}^{(m)}}=\frac{|\partial E / \partial m|}{\left|\partial E / \partial n_{r}\right|}=\left(\frac{|m| \hbar}{\pi \sqrt{2 \mu E}}\right)\left[\int_{R_{\min }}^{R} \frac{d r}{r \sqrt{r^{2}-R_{\min }^{2}}}\right] .
$$

If we evaluate the integral and use $R_{\min } \equiv|m| \hbar / \sqrt{2 \mu E}$, we find that

$$
\frac{q}{p}=\frac{1}{\pi} \sec ^{-1}\left(\frac{R}{R_{\min }}\right)
$$

or 


$$
R_{\min }(p, q) \equiv R_{\min }=R \cos \left(\frac{\pi q}{p}\right)
$$

as the condition on periodic orbits. To find the classical period for such closed orbits, we note that

$$
T_{\mathrm{cl}}^{(\mathrm{po})}=p T_{\mathrm{cl}}^{\left(n_{r}\right)}=p\left[\frac{2 \pi \hbar}{\left|\partial E / \partial n_{r}\right|}\right]=\left(2 p \sqrt{R^{2}-R_{\min }^{2}}\right) \sqrt{\frac{\mu}{2 E}}=\frac{2 p R \sin (\pi q / p)}{v_{0}}=\frac{L(p, q)}{v_{0}},
$$

where we identify $v_{0}=\sqrt{2 E / \mu}$ with the classical speed. The condition in Eq. (779) and the resulting path lengths/periods in Eq. (80) can be obtained from strictly geometrical $\operatorname{arguments.~}^{38.50}$ Just as for the square and equilateral triangle cases, information on the classical closed orbits is clearly encoded in the energy eigenvalue spectrum for the circular well using Eqs. (32) and (34). The values of these path lengths, $L(p, q)=2 p R \sin (\pi q / p)$, and the corresponding distances of closest approach, $R_{\min } / R=R \cos (\pi q / p)$, for many of the lowest-lying closed or periodic orbits in the circular well are collected in Table II.

\section{B. Wave packet construction in the circular billiard}

To examine the construction of Gaussian wave packets in this geometry, we note that any arbitrary initial wave packet, $\psi(r, \theta)$, in the circular billiard can be expanded in the normalized eigenstates of the form

$$
w_{\left(m, n_{r}\right)}(r, \theta)=\left[N_{\left(m, n_{r}\right)} J_{|m|}\left(k_{\left(m, n_{r}\right)} r\right)\right]\left(\frac{1}{\sqrt{2 \pi}} e^{i m \theta}\right)
$$

where

$$
\left[N_{\left(m, n_{r}\right)}\right]^{2} \int_{0}^{R} r\left[J_{|m|}(k r)\right]^{2} d r=1
$$

with expansion coefficients given by

$$
a_{\left(m, n_{r}\right)}=\left\langle\psi(r, \theta ; t=0) \mid w_{\left(m, n_{r}\right)}\right\rangle
$$

which satisfy

$$
\sum_{m=-\infty}^{+\infty} \sum_{n_{r}=0}^{\infty}\left|a_{\left(m, n_{r}\right)}\right|^{2}=1
$$

Because the eigenstates are no longer simple trigonometric functions, the required normalization and overlap integrals must be done numerically, making the process of wavepacket 
construction much more involved. The expectation value of the energy in this potential well is given by

$$
\langle\hat{E}\rangle=\sum_{m=-\infty}^{+\infty} \sum_{n_{r}=0}^{\infty}\left|a_{\left(m, n_{r}\right)}\right|^{2} E_{\left(m, n_{r}\right)}=\sum_{m=-\infty}^{+\infty} \sum_{n_{r}=0}^{\infty}\left|a_{\left(m, n_{r}\right)}\right|^{2}\left(\frac{\hbar^{2}\left[z_{\left(m, n_{r}\right)}\right]^{2}}{2 \mu R^{2}}\right),
$$

and this constraint is another useful one for numerical checks.

In this more symmetrical geometry, we also can evaluate expectation values of powers of the angular momentum, $\hat{L}_{z}^{k}$, which is the other important conserved quantity. We find that

$$
\left\langle\hat{L}_{z}^{k}\right\rangle=\sum_{m=-\infty}^{+\infty} \sum_{n_{r}=0}^{\infty}\left|a_{\left(m, n_{r}\right)}\right|^{2}(m \hbar)^{k}
$$

The subsequent time-dependence of any such wave packet is then given by

$$
\psi(r, \theta ; t)=\sum_{m=-\infty}^{+\infty} \sum_{n_{r}=0}^{\infty} a_{\left(m, n_{r}\right)} w_{\left(m, n_{r}\right)}(r, \theta) e^{-i E_{\left(m, n_{r}\right)} t / \hbar}
$$

and the autocorrelation function is given by ${ }^{33}$

$$
A(t) \equiv\langle\psi(r, \theta ; t) \mid \psi(r, \theta, 0)\rangle=\sum_{m=-\infty}^{+\infty} \sum_{n_{r}=0}^{\infty}\left|a_{\left(m, n_{r}\right)}\right|^{2} e^{-i E_{\left(m, n_{r}\right)} t / \hbar} .
$$

We now focus on the specific Gaussian form in Eq. (43), besides the expectation values calculated above for such a form. We can explicitly evaluate the conserved powers of the angular momentum, namely

$$
\left\langle\hat{L}_{z}\right\rangle=\left\langle x \hat{p}_{y}-y \hat{p}_{x}\right\rangle=\langle x\rangle\left\langle\hat{p}_{y}\right\rangle-\langle y\rangle\left\langle\hat{p}_{x}\right\rangle=x_{0} p_{0 y}-y_{0} p_{0 x}
$$

and

$$
\left\langle\hat{L}_{z}^{2}\right\rangle=\left(x_{0} p_{0 y}-y_{0} p_{0 x}\right)^{2}+\frac{b^{2}}{2}\left[\left(p_{0 x}\right)^{2}+\left(p_{0 y}\right)^{2}\right]+\frac{\hbar^{2}}{2 b^{2}}\left[\left(x_{0}\right)^{2}+\left(y_{0}\right)^{2}\right],
$$

so that the spread (or uncertainty) in the angular momentum is given by

$$
(\Delta m) \hbar \equiv \Delta L_{z}=\sqrt{\frac{b^{2}}{2}\left[\left(p_{0 x}\right)^{2}+\left(p_{0 y}\right)^{2}\right]+\frac{\hbar^{2}}{2 b^{2}}\left[\left(x_{0}\right)^{2}+\left(y_{0}\right)^{2}\right]}
$$

This form can be understood intuitively from a simple error propagation argument that would give the error in the product $L \sim r p$ to be $(\Delta L / L)^{2}=(\Delta r / r)^{2}+(\Delta p / p)^{2}$ or

$$
\Delta L=\sqrt{p^{2}(\Delta r)^{2}+r^{2}(\Delta p)^{2}}
$$


We note that even for wavepackets for which the expectation value of the angular momentum vanishes, it may still be necessary to include $m \neq 0$ components; for example, a wave packet with $\left(x_{0}, y_{0}\right)=(0,0)$ and $\left(p_{0 x}, p_{0 y}\right)=\left(0, p_{0}\right)$, will have a necessary spread in angular momentum values given by $\Delta m=b p_{0} / \sqrt{2} \hbar$, which increases linearly with the initial momentum. For such cases, because $\left\langle\hat{L}_{z}\right\rangle=0$, we must have relations such as $\left|a_{\left(m, n_{r}\right)}\right|=\left|a_{\left(-m, n_{r}\right)}\right|$ to ensure that Eq. (86) is satisfied.

Because of the required numerical evaluation of the expansion coefficients, it is useful to be able to compare the general results for $\langle E\rangle$ and $\left\langle\hat{L}_{z}^{(1,2)}\right\rangle$ in Eqs. (85) and (86) with the specific results for the Gaussian in Eqs. (45), (89), and (90). As an example of such an expansion, we have numerically evaluated the $a_{\left(m, n_{r}\right)}$ for Gaussian wave packets characterized by $y_{0}, p_{0 x}=0$, $p_{0 y}=100$, and $x_{0} / R=0.0,-0.5$ and 0.70 , that is, with the same total (kinetic) energy, but different values of the angular momentum; the same physical parameters of Eq. (25) are used, along with $R=1$. The various expansion probabilities, $\left|a_{\left(m, n_{r}\right)}\right|^{2}$, are sorted by magnitude, and then summed to confirm that the numerical evaluation has captured all of the probability density. We can then illustrate which regions of the $\left(m, n_{r}\right)$ space are most populated in the construction of each wave packet and the results are shown in Fig. 5. In each case, the (approximately elliptical) inner shaded areas correspond to regions in $\left(m, n_{r}\right)$ space containing 68\% (inner dark region) of the total probability, while the outer dark regions surround $99.7 \%$ of the $\sum_{\left(m, n_{r}\right)}\left|a_{\left(m, n_{r}\right)}\right|^{2}$. We note that the required spread in $m$ values increases for those states with increasing values of $|\langle\hat{L}\rangle|=x_{0} p_{0 y}$.

We can also follow the short-time development of Gaussian wave packets in the circular billiard for various initial conditions to look for evidence of the classical periodicities described by Eqs. (79) and (80). In this case we construct packets with $y_{0}=0, p_{0 x}=0$, $p_{0 y}=100$ and various values of $x_{0}=R_{\text {min }}$. (We use a much smaller value of $p_{0}$ than in earlier calculations for the square well because we are restricted to evaluating the expansion coefficients numerically which becomes prohibitively time-consuming for larger values: the repeated evaluation of high-order Bessel functions is numerically intensive in the programs we use and is the limiting factor. This unavoidable restriction means that the spreading time, $t_{0}$, is of the same order as the classical periods shown.) We plot in Fig. 6 the autocorrelation function $|A(t)|^{2}$ versus $t / \tau$, where $\tau \equiv R / v_{0}$. We note that isolated features in $A(t)$ are present at the values of $T_{\mathrm{cl}}^{(\mathrm{po})} / \tau=2 p \sin (\pi q / p)$ and $R_{\min } / R=\cos (\pi q / p)$ values given in Table II, corresponding to classical closed orbits in this geometry. 
We can extend this analysis to the half-circle billiard, obtained by folding across a diameter in the same way as we have done for the half-square and half-equilateral triangle. In this case, we use a single copy of the $m>0$ eigenstates. 31

\section{CONCLUDING REMARKS}

We have examined the quantum mechanical time-development of localized (Gaussianlike) wave packets in three billiard systems, with square, equilateral triangle, and circular footprints. We have used the well-known (and not-so-well-known) energy eigenvalue spectrum in each case to discuss the classical periodicity and quantum revival time scales in such systems using the autocorrelation function as a probe. In the first two cases, we used the corresponding energy eigenfunctions to calculate closed form, analytical approximations for the expansion coefficients of the Gaussian wave packets, suitable for fast numerical evaluations for arbitrarily large momentum values, provided the initial wave packets are well-localized within the well and reasonably far from any infinite wall boundary. For the circular well, we have performed a similar analysis, using a WKB analysis to demonstrate the expected short-time classical periodicities and a numerical approach to evaluate $A(t)$.

In each case, we have also been able to consider half-well geometries, obtained by folding of the original footprint along an obvious axis of symmetry for which solutions were available as a subset of those for the full-well.

Further extensions of these studies could include more detailed examinations of rectangular geometries, or even three-dimensional (3D) parallelepipeds. The case of an annular billiard (with both outer and inner infinite walls) $\underline{\underline{49}}$ can be handled in much the same way as described here for the 2D circular well, and the same WKB approach can be used to extract the pattern of allowed classical closed orbits, which is much richer than in the simple circular billiard.

The spherical billiard also can be discussed with many of the same techniques. The energy eigenstates now consist of products of spherical harmonics, $Y_{l, m}(\theta, \phi)$, instead of the simple $e^{i m \theta}$ angular states, with spherical Bessel functions for the radial component. We might expect that the resulting change in the angular momentum term from two to three dimensions would be given by the substitution $m^{2} \rightarrow l(l+1)$, but it turns out that

for a WKB analysis it is more appropriate ${ }^{48}$ to use $(l+1 / 2)^{2}$ instead of $l(l+1)$ (the so- 
called Langer modification. $\left.{ }^{51}\right)$ This identification still gives the same result for the classical periods, which is not surprising because angular momentum conservation implies that all the allowed 3D classical orbits will be planar and reduce to the 2D case. This effect can be seen rather generally in 3D central potential systems where we know that the quantized energy eigenvalues do not depend on the $m$ quantum number, namely the $E\left(n_{r}, l, m\right)=E\left(n_{r}, l\right)$ are functions of $n_{r}$ and $l$ alone. This independence implies that the classical periodicity corresponding to the $m$ quantum number in Eq. (32) will be $T_{\mathrm{cl}}^{(m)}=2 \pi \hbar /|\partial E / \partial m| \rightarrow \infty$ which becomes irrelevant, leaving only $T_{\mathrm{cl}}^{\left(n_{r}\right)}$ and $T_{\mathrm{cl}}^{(l)}$ to beat against each other to provide closed orbits.

In the context of the spherical billiard, we note that the spherical Bessel functions, $j_{l}(z)$, can be related to the ones considered here for the $2 \mathrm{D}$ case via $j_{l}(z)=\sqrt{\pi / 2 z} J_{l+1 / 2}(z)$. For purely central (no angular momentum, s-wave) Gaussian wave packets (therefore with initial position at $\left(x_{0}, y_{0}, z_{0}\right)=(0,0,0)$ and with vanishing momentum), the required $l=0$ spherical Bessel function is proportional to $\sin (z) / z$, which does have exact integral values of $z=k R=n \pi$. For this case only, there will be exact quantum revivals in the spherical billiard.

The solutions for the equilateral triangle case $(N=3$ regular polygon) form a subset of those required for a complete study of the $(N=6)$ hexagonal billiard and that case is currently under investigation. It is an interesting and open question as to whether there are other $N$ values (besides $N=3$ and 4) for which there exist exact quantum revivals as well as well as how the $N \rightarrow \infty$ limit of the circular billiard (where only approximate revivals are present) is reached. Approximation methods appropriate for such quantum billiard systems ${ }^{52}$ may be useful in answering such questions.

We also can extend the notions explored here dealing with classical periodicity to nonbilliard systems. For example, the extension of Eqs. (32) and (34) to three dimensions is obvious. As mentioned, for central potentials the $T_{\mathrm{cl}}^{(m)}$ classical period is irrelevant, so that closed or periodic orbits are the result of the condition $p T_{\mathrm{cl}}^{\left(n_{r}\right)}=T_{\mathrm{cl}}^{(\mathrm{po})}=q T_{\mathrm{cl}}^{(l)}$ for the $\left(n_{r}, l\right)$ quantum numbers. It is a familiar result of classical mechanics (Bertrand's theorem 53.54 ) that for the 3D power law potentials $\left(V(r) \propto r^{k}\right)$, only the harmonic oscillator $(k=2)$ and Coulomb potentials $(k=-1)$ give rise to closed orbits for all bound state trajectories. ${ }^{53}$ It is straightforward to exhibit a similar statement for the quantum versions of these systems in terms of their expected classical periods, $T_{\mathrm{cl}}^{(p o)}$. 


\section{Acknowledgments}

The work of R. W. R. was supported, in part, by the National Science Foundation under Grant DUE-9950702. The work of M. A. D. was supported, in part, by the Commonwealth College of the Pennsylvania State University under a Research Development Grant (RDG).

\section{APPENDIX A: WKB ANALYSIS OF CLASSICAL PERIODS}

As a comparison with the formalism leading to Eq. (15), an alternative derivation of the expression for the classical periodicity of a bound state system in terms of its quantized energy levels can be obtained from a simple WKB argument. For a particle of fixed energy $\mathrm{E}$ in a bound state potential, $V(x)$, we have $E=\mu v(x)^{2} / 2+V(x)$ and the short time, $d t$, required to traverse a distance $d x$ can be obtained from this energy conservation connection and integrated over a single cycle to obtain the classical period via

or

$$
d t=\sqrt{\frac{\mu}{2}} \frac{d x}{\sqrt{E-V(x)}},
$$

$$
\tau=2 \int_{a}^{b} d t=2 \sqrt{\frac{\mu}{2}} \int_{a}^{b} \frac{d x}{\sqrt{E-V(x)}} .
$$

The WKB quantization condition for this potential can be written in the form $\underline{48}$

$$
\sqrt{2 \mu} \int_{a}^{b} \sqrt{E_{n}-V(x)} d x=\left(n+C_{L}+C_{R}\right) \pi \hbar
$$

in terms of the matching coefficients $C_{L}$ and $C_{R}$. Recall that $C_{L, R}=1 / 4$ at linear or smooth turning points where the functions are matched smoothly onto Airy solutions, while $C_{L, R}=1 / 2$ at infinite wall type boundaries where the wavefunction must vanish. ${ }^{48}$ This difference is sometimes described as being due to the fact that WKB wavefunctions can penetrate roughly one-eighth of a wavelength into the classically disallowed region, provided the barrier is not infinitely high. .55

Equation (A2) can be differentiated implicitly with respect to the quantum number $n$ to obtain

$$
\sqrt{2 \mu} \int_{a}^{b} \frac{\left|d E_{n} / d n\right| d x}{2 \sqrt{E_{n}-V(x)}}=\pi \hbar .
$$

Equation (A3) can be related to the classical period in Eq. (A1b) to give

$$
\tau_{n} \equiv \sqrt{2 \mu} \int_{a}^{b}\left(E_{n}-V(x)\right)^{-1 / 2} d x=\frac{2 \pi \hbar}{\left|d E_{n} / d n\right|},
$$


as in Eq. (15). The most obvious example of such a connection is for the harmonic oscillator, where the WKB condition gives the exact eigenvalues, $E_{n}=(n+1 / 2) \hbar \omega$, and the classical period from Eq. (A4) is

$$
\tau_{n}=\frac{2 \pi \hbar}{|d E / d n|}=\frac{2 \pi \hbar}{\hbar \omega}=\frac{2 \pi}{\omega}
$$

as expected.

\section{APPENDIX B: EXPANSION COEFFICIENTS FOR THE 1D INFINITE WELL IN MOMENTUM SPACE}

Although it is typical to discuss the expansion coefficients for a Gaussian wave packet in the 1D infinite well in position space, as in Eqs. (17) or (26), it is instructive to examine the same problem in momentum space. As mentioned in Sec. III the requirement of the necessary matching of the oscillatory $e^{-i p_{x} x / \hbar}$ factor in $\psi_{G}(x, 0)$ with the corresponding $e^{ \pm i n \pi x / a}$ factors from the bound state $u_{n}(x)$ is contained explicitly in the $\exp \left(-b^{2}\left(p_{0} \pm\right.\right.$ $n \pi \hbar / a)^{2} / 2 \hbar^{2}$ ) terms in Eq. (26). There must also be an appropriate overlap between the envelope of $\psi_{G}(x, 0)$ contained in the $\exp \left(-\left(x-x_{0}\right)^{2} / 2 b^{2}\right)$ factor and the extent of the bound state wavefunctions. That is, it must be inside the well, as illustrated in Fig. 1.

The form of the momentum-matching can be qualitatively understood in the following way. The oscillatory parts of the integrals required for the evaluation of $a_{n}$ will contain factors such as $\exp \left(i\left(p_{0} \pm n \pi \hbar / a\right) x / \hbar\right)$ to be integrated over $x$. An integral over all space would give rise to a $\delta$-function term of the form $\delta\left(\left[p_{0} \pm n \pi \hbar / a\right] / \hbar\right)$, because of the cancellations arising from the rapid oscillations of the integrand. However, the integrals are effectively cut off by the spatial extent of the initial wave packet, a width of order $\Delta x \sim b$, which instead gives a sharply peaked function of $\left|p_{0} \pm n \pi \hbar / a\right| b / \hbar$, as seen in Eq. (26).

The same information must be encoded in a momentum space representation of the evaluation of the $a_{n}$ expansion coefficients. The required Fourier transform

$$
\phi_{n}(p)=\frac{1}{\sqrt{2 \pi \hbar}} \int_{0}^{a} u_{n}(x) e^{-i p x / \hbar} d x
$$

of the bound-state wavefunction $u_{n}(x)=\sqrt{\frac{2}{a}} \sin \left(\frac{n \pi x}{a}\right)$ is given by

$$
\begin{aligned}
\phi_{n}(p) & =-\frac{a}{\sqrt{4 \pi \hbar a}}\left[\frac{e^{i(n \pi-p a / \hbar)}-1}{(n \pi-p a / \hbar)}+\frac{e^{-i(n \pi+p a / \hbar)}-1}{(n \pi+p a / \hbar)}\right] \\
& =-\frac{a i}{\sqrt{4 \pi \hbar a}}\left[e^{i n \pi} \frac{\sin [(n \pi-p a / \hbar) / 2]}{[(n \pi-p a / \hbar) / 2]}-e^{-i n \pi} \frac{\sin [(n \pi+p a / \hbar) / 2]}{[(n \pi+p a / \hbar) / 2]}\right] e^{-i p a / 2 \hbar}
\end{aligned}
$$


while the initial Gaussian from Eq. (11) is

$$
\phi_{G}(p)=\sqrt{\frac{\alpha}{\sqrt{\pi}}} e^{-\alpha^{2}\left(p-p_{0}\right)^{2} / 2} e^{-i p x_{0} / \hbar} .
$$

The (exact) expansion coefficients now have the form

$$
a_{n}=\int_{-\infty}^{+\infty} \phi_{n}^{*}(p) \phi_{G}(p, 0) d p
$$

The matching in momentum space now comes from the requirement that the peak in $\phi_{G}(p)$ (near $p_{0}$ ) must match with those in $\phi_{n}(p)$ (near $p= \pm n \pi \hbar / a$ ). The information on whether the initial wave packet is contained inside the $(0, a)$ infinite well now arises from the phase information contained in the oscillatory parts of the momentum space versions: specifically, the $a_{n}$ integral in Eq. (B4) contains terms such as

$$
\left[\phi_{n}^{*}(p)\right]\left[\phi_{G}(p, 0)\right] \propto\left[e^{-i p a / 2 \hbar}\right]^{*}\left[e^{-i p x_{0} / \hbar}\right]=e^{i p / \hbar\left(a / 2-x_{0}\right)},
$$

which, if integrated by itself over all $p$-space, would give rise to a $\delta\left(x_{0}-a / 2\right)$ term. However, this singular behavior is again softened to be a sharply peaked function of $\left|x_{0}-a / 2\right|$, due to the finite extent of the $p$-integrals. It should not be surprising that the roles played by the envelope/overlap requirement versus that played by the oscillatory information are complementary in the $x$ - versus $p$-space descriptions of the integrals in Eqs. (24) and (B4)

We can easily generalize this last result to the case of an infinite well of width $a$, but located at an arbitrary location along the 1D axis defined over the range $(d, d+a)$. In that case, the energies are unchanged, and the position space eigenfunctions are simply shifted to

$$
\tilde{u}_{n}(x)=u_{n}(x-d)=\sqrt{\frac{2}{a}} \sin \left(\frac{n \pi(x-d)}{a}\right) .
$$

The Fourier transform to obtain the corresponding momentum space eigenstates goes through as before with a simple change of integration variables, $x-d \rightarrow y$, giving

$$
\tilde{\phi}_{n}(p)=\phi_{n}(p) e^{-i p d / \hbar}
$$

in which case the appropriate phase factor, analogous to that in Eq. (ㅍ5) , is $\exp (i p / \hbar(d+$ $\left.a / 2-x_{0}\right)$ ); values of $x_{0}$ beyond the boundaries of the new well lead to exponential suppression.

\section{APPENDIX C: PROBLEMS}

We offer some suggested problems that are motivated by the suggestions in Sec. VII. 
Problem 1. Extend the results of Sec. IVB for a square to a rectangular billiard with sides $L_{x} \times L_{y}$. Calculate the allowed energies and the classical periods. Discuss the patterns of exact and/or fractional revivals. (See Refs. 35 and 36 for details.)

Problem 2. Extend the relationship in Eq. (34) to a system with three quantum numbers to find the allowed classical periods. Apply this extension of Eq. (34) to the system of a cubical box of side $L$, generalizing the result of Eq. (42).

Problem 3. Visualize some of the closed orbits for the equilateral triangle geometry. (See Ref. 32 for examples.)

Problem 4. Generalize the WKB results of Sec. VIA to show how the closed orbits (periods and path lengths) in an annular infinite well are obtained. How is Eq. (766) changed, including the limits of integration and the matching coefficients, $C_{L}$ and $C_{R}$ ? (See Ref. 49 for details.)

Problem 5. Plot the equivalent of Fig. 5 for Gaussian wave packets in a square well, initially centered at the origin, with initial momentum components $\left(p_{0 x}, p_{0 y}\right)=\left(p_{0} \cos (\theta), p_{0} \sin (\theta)\right)$ for various values of $\theta$. That is, evaluate the expansion coefficients, $a_{\left(n_{x}, n_{y}\right)}=a_{n_{x}} a_{n_{y}}$, and the corresponding probabilities, and plot where the probability is distributed in the $\left(n_{x}, n_{y}\right)$ plane. 


\section{TABLES}

\begin{tabular}{|r|c|r||r|c|c|}
\hline$\theta(\mathrm{deg})$ & period $T_{\mathrm{cl}}^{(\mathrm{po})} / \tau$ & $(p, q)$ & $\theta(\mathrm{deg})$ & period $T_{\mathrm{cl}}^{(\mathrm{po})} / \tau$ & $(p, q)$ \\
\hline 0.00 & $1.00,2.00,3.00, \ldots$ & $(1,0)$ & 23.96 & 9.85 & $(9,4)$ \\
\hline 6.34 & 9.06 & $(9,1)$ & 26.57 & $2.24,4.47,6.71,8.94$ & $(2,1)$ \\
\hline 7.13 & 8.06 & $(8,1)$ & 29.74 & 8.06 & $(5,3)$ \\
\hline 8.13 & 7.07 & $(7,1)$ & 30.96 & 5.83 & $(7,4)$ \\
\hline 9.46 & 6.08 & $(6,1)$ & 32.00 & 9.43 & $(8,5)$ \\
\hline 11.31 & 5.10 & $(5,1)$ & 33.69 & $3.61,7.21$ & $(3,2)$ \\
\hline 12.53 & 9.22 & $(9,2)$ & 35.54 & 8.60 & $(7,5)$ \\
\hline 14.04 & $4.12,8.25$ & $(4,1)$ & 36.87 & $5.00,10.00$ & $(4,3)$ \\
\hline 15.95 & 7.28 & $(7,2)$ & 38.66 & 6.40 & $(5,4)$ \\
\hline 18.43 & $3.16,6.32,9.49$ & $(3,1)$ & 39.81 & 7.81 & $(6,5)$ \\
\hline 20.56 & 8.54 & $(8,3)$ & 40.60 & 9.22 & $(7,6)$ \\
\hline 21.80 & 5.38 & $(5,2)$ & 45.00 & $1.41,2.83,4.24, \ldots$ & $(1,1)$ \\
\hline 23.20 & 7.62 & $(7,3)$ & & & \\
\hline
\end{tabular}

Table I: The periods for the classical closed orbits, $T_{\mathrm{cl}}^{(\mathrm{po})} / \tau=\sqrt{p^{2}+q^{2}}$, where $\tau \equiv 2 a / v_{0}$, and the corresponding initial angles, $\tan (\theta)=q / p$, for the square billiard. All periodic orbits with $T_{\mathrm{cl}}^{(p o)} / \tau \leq 10$ are included. Values for $45^{\circ}<\theta<90^{\circ}$ are the same as those for $90^{\circ}-\theta$. 


\begin{tabular}{|c|c|c||c|c|c|}
\hline$(p, q)$ & $L / R=2 p \sin (\pi q / p)$ & $\begin{array}{c}R_{\min } / R= \\
\cos (\pi q / p)\end{array}$ & $(p, q)$ & $L / R=2 p \sin (\pi q / p)$ & $\begin{array}{l}R_{\min } / R= \\
\cos (\pi q / p)\end{array}$ \\
\hline$(2,1)$ & $4.00,8.00,12.00,16.00, \ldots$ & 0.00 & $(6,3)$ & $12.00, \ldots$ & 0.00 \\
\hline$(3,1)$ & $5.20,10.39, \ldots$ & 0.50 & $(7,3)$ & $13.65, \ldots$ & 0.22 \\
\hline$(4,1)$ & $5.66,11.31, \ldots$ & 0.71 & $(8,3)$ & $14.78, \ldots$ & 0.38 \\
\hline$\vdots$ & $\vdots$ & $\vdots$ & $(9,3)$ & $15.59, \ldots$ & 0.50 \\
\hline$(\infty, 1)$ & $6.28,12.57, \ldots$ & 1.00 & $(10,3)$ & $16.18, \ldots$ & 0.59 \\
\hline & & & $(11,3)$ & $16.63, \ldots$ & 0.66 \\
\hline$(4,2)$ & $8.00,16.00, \ldots$ & 0.00 & $(12,3)$ & $16.97, \ldots$ & 0.71 \\
\hline$(5,2)$ & $9.51, \ldots$ & 0.31 & $(13,3)$ & $17.24, \ldots$ & 0.75 \\
\hline$(6,2)$ & $10.39, \ldots$ & 0.50 & $\vdots$ & $\vdots$ & $\vdots$ \\
\hline$(7,2)$ & $10.95, \ldots$ & 0.62 & $(\infty, 3)$ & $18.85, \ldots$ & 1.00 \\
\hline$\vdots$ & $\vdots$ & $\vdots$ & & & \\
\hline$(\infty, 2)$ & $12.57, \ldots$ & 1.00 & & & \\
\hline
\end{tabular}

Table II: Tabulated values of the path length, $L / R=2 p \sin (\pi q / p)$, from Eq. (80) and the distance of closest approach, $R_{\min } / R=\cos (\pi q / p)$, from Eq. (79) for low-lying closed or periodic orbits for the circular well characterized by $(p, q)$. All closed orbits with path lengths satisfying $L(p, q)<20 R$ are included, as are their low-lying recurrences. 
* Electronic address: mad10@psu.edu

$\dagger$ Electronic address: rick@phys.psu.edu

1 For an excellent review of wave packet dynamics, see The Physics and and Chemistry of Wave Packets, edited by J. A. Yeazell and T. Uzer (Wiley, NY, 2000).

2 J. R. Hiller, I. D. Johnston, and D. F. Styer, Quantum Mechanics Simulations: The Consortium for Upper-Level Physics Software (Wiley, NY, 1995); B. Thaller, Visual Quantum Mechanics: Selected Topics with Computer-Generated Animations of Quantum-Mechanical Phenomena (Springer-Verlag, NY, 2000); S. Brandt and S. Dahmen, The Picture Book of Quantum Mechanics (Springer-Verlag, NY, 2001), 3rd ed.; J. Bayfield, Quantum Evolution: An Introduction to Time-Dependent Quantum Mechanics (Wiley, NY, 1999); S. M. McMurry, Quantum Mechanics (Addison-Wesley, Reading, MA, 1993); J. Jarecki, Graphical Schrödingers Equation, (Physics Academic Software, Raleigh, 1998); W. Christian and M. Belloni, Physlets: Teaching Physics with Interactive Curricular Material (Prentice-Hall, Englewood Cliffs, 2000).

3 C. Singh, "Student understanding of quantum mechanics," Am. J. Phys. 69, 885-895 (2001).

4 E. Cataloglu and R. W. Robinett, "Testing the development of student conceptual and visualization understanding in quantum mechanics through the undergraduate career," Am. J. Phys. 70, 238-251 (2002).

5 A. Goldberg, H. M. Schey, and J. L. Schwartz, "Computer-generated motion pictures of onedimensional quantum-mechanical transmission and reflection phenomena," Am. J. Phys. 35, 177-186 (1967).

6 M. H. Bramhall and B. M. Casper, "Reflections on a wave packet approach to quantum mechanical barrier penetration," Am. J. Phys. 38, 1136-1145 (1970).

7 B. Diu, "Plane waves and wave packets in elementary quantum mechanics problems," Eur. J. Phys. 1, 231-240 (1980).

8 A. Edgar, "Reflection of wave packets from a quantum well with a tunneling transmission resonance," Am. J. Phys. 63, 136-141 (1995).

9 J. S. Boleman and S. B. Haley, "More time-dependent calculations for the Schrödinger equation," Am. J. Phys. 43, 270-271 (1975).

10 M. Andrews, "Wave packets bouncing off walls," Am. J. Phys. 66, 252-254 (1998). 
11 M. A. Doncheski and R. W. Robinett, "Anatomy of a quantum bounce," Eur. J. Phys. 20, 29-37 (1999).

12 J. V. Greenman, "Non-dispersive mirror wave packets," Am. J. Phys. 40, 1193-1201 (1972).

13 C. U. Segre and J. D. Sullivan, "Bound-state wave packets," Am. J. Phys. 44, 729-732 (1976). This paper also contains one of the first references to what have become known as wave packet revivals.

14 P. A. Deutchmann, "Tunneling between two square wells - Computer movie," Am. J. Phys. 39, 952-954 (1971).

15 E. A. Johnson and H. Thomas Williams, "Quantum solutions for a symmetric double square well," Am. J. Phys. 50, 239-243 (1981).

16 J. C. Hamilton, J. L. Schwartz, and W. A. Bowers, "Computer generated films for solid state physics," Am. J. Phys. 40, 1657-1972 (1972).

17 G. Friedmann and W. A. Little, "A study of a wave function of a particle striking a crystal interface," Am. J. Phys. 61, 835-843 (1993).

18 J. R. Merrill, "The propagation of quantum mechanical wave packets," Am. J. Phys. 41, 1101$1103(1973)$.

19 R. Bluhm, V. A. Kostelecký, and J. Porter, "The evolution and revival structure of localized wave packets," Am. J. Phys. 64, 944-953 (1996).

20 R. W. Robinett, "Visualizing the collapse and revival of wave packets in the infinite square well using expectation values," Am. J. Phys, 68, 410-420 (2000).

21 D. F. Styer, "Quantum revivals versus classical periodicity in the infinite square well," Am. J. Phys. 69, 56-62 (2001).

22 F. Großmann, J. -M. Rost, and W. P. Schleich, "Spacetime structures in simple quantum systems," J. Phys. A30, L277-L283 (1997).

23 P. Stifter, W. E. Lamb Jr, and W. P. Schleich, "The particle in a box revisited," in Frontiers of Quantum Optics and Laser Physics, Proceedings of the International Conference on Quantum Optics and Laser Physics (Springer, Singapore, 1997) edited by S. Y. Zhu, M. S. Zubairy, and M. O. Scully, pp. 236-246.

24 J. Gea-Banacloche, "A quantum bouncing ball," Am. J. Phys. 67, 776-782 (1999).

25 M. A. Doncheski and R. W. Robinett, "Expectation value analysis of wave packet solutions for the quantum bouncer: Short-term classical and long-term revival behaviors," Am. J. Phys. 69, 
1084-1090 (2001).

26 D. L. Aronstein and C. R. Stroud Jr, "Fractional wave-function revivals in the infinite square well," Phys. Rev. A55, 4526-4537 (1997).

27 K. Razi Naqvi, S. Waldenstrom, and T. Jaji Hassan, "Fractional revival of wave packets in an infinite square well: A Fourier perspective," Eur. J. Phys. 22, 395-402 (2001).

28 W. Loinaz and T. J. Newman, "Quantum revivals and carpets in some exactly solvable systems," J. Phys. A: Math. Gen. 32, 8889-8895 (1999).

29 C. M. Marcus, A. J. Rimberg, R. M. Westervelt, P. F. Hopkins, and A. C. Gossard, "Conductance fluctuations and chaotic scattering in ballistic microstructures," Phys. Rev. Lett. 69, 506-509 (1992).

30 N. Friedman, A. Kaplan, D. Carasso, and N. Davison, "Observation of chaotic and regular dynamics in atom-optics billiards," Phys. Rev. Lett. 86, 1518-1521 (2001).

31 R. W. Robinett and S. Heppelmann, "Quantum wave packet revivals in circular billiards," Phys. Rev. A65, 062103-062113 (2002).

32 M. A. Doncheski and R. W. Robinett, "Quantum mechanical analysis of the equilateral triangle billiard: periodic orbit theory and wave packet revivals," Ann. Phys. (NY) 299, 208-227 (2002).

33 M. Nauenberg, "Autocorrelation function and quantum recurrence of wave packets," J. Phys. B. 23, L385-L390 (1990).

34 R. W. Robinett, Quantum Mechanics: Classical Results, Modern Systems, and Visualized Examples (Oxford University Press, NY, 1997), pp. 142-143.

35 R. Bluhm, V. Alan Kostelecký, and B. Tudose, "Wave-packet revivals for quantum systems with nondegenerate energies," Phys. Lett. A 222, 220-226 (1996).

36 G. S. Agarwal and J. Banerji, "Fractional revivals in systems with two time scales," Phys. Rev. A 57, 3880-3884 (1998).

37 K. Richter, D. Ullmo, and R. A. Jalabert, "Orbital magnetism in the ballistic regime: geometrical effects," Phys. Rep. 276, 1-83 (1996)

38 R. W. Robinett, "Visualizing classical periodic orbits from the quantum energy spectrum via the Fourier transform: Simple infinite well examples," Am. J. Phys. 65, 1167-1175 (1997).

39 M. V. Berry and M. Wilkinson, "Diabolical points in the spectra of triangles," Proc. Royal Society, London A 392, 15-43 (1984).

40 W. -K. Li, “A particle in an isosceles right triangle," J. Chem. Educ. 61, 1034 (1984). 
41 R. W. Robinett, "Isolated versus non-isolated periodic orbits in variants of the two-dimensional square and circular billiards," J. Math. Phys. 40, 101-122 (1999).

42 C. Jung, "An exactly soluble three-body problem in one-dimension," Canadian J. Phys. 58, 719-728 (1980). This paper discusses the group theoretical properties of the solutions of the triangular well in some detail.

43 P. J. Richens and M. V. Berry, "Pseudointegrable systems in classical and quantum mechanics," Physica D 2, 495-512 (1981).

44 A version of this problem is also discussed in J. Mathews and R. L. Walker, Mathematical Methods of Physics (W. A. Benjamin, Menlo Park, 1970), 2nd ed., pp. 237-239.

45 W. -K. Li and S. M. Blinder, "Particle in an equilateral triangle: Exact solution of a nonseparable problem," J. Chem. Educ. 64, 130-132 (1987).

46 I. Galbraith, Y. S. Ching, and E. Abraham, "Two-dimensional time-dependent quantummechanical scattering event," Am. J. Phys. 52, 60-68 (1984).

47 See, for example, M. Abramowitz and I. A. Stegun, Handbook of Mathematical Functions (McGraw-Hill, NY, 1964), p. 364.

48 A. B. Migdal and V. Krainov, Approximation Methods in Quantum Mechanics (Benjamin, NY, 1992), pp. 106-115.

49 S. M. Reimann, M. Brack, A. G. Magner, and M. V. N. Murthy, "Applications of classical periodic orbit theory to circular billiards with small scattering centers," Surf. Rev. Lett. 3, 19-23 (1996); R. W. Robinett, "Energy eigenvalues and periodic orbits for the circular disk or annular infinite well," Surf. Rev. Lett. 5, 519-526 (1998); R. W. Robinett, "Periodic orbit theory analysis of the circular disk or annular billiard," Am. J. Phys. 67, 67-77 (1999).

50 R. Balian and C. Bloch, "Distribution of eigenfrequencies for the wave equation in a finite domain III. Eigenfrequency density oscillations," Ann. Phys. 69, 76-160 (1972).

51 For a discussion and references, see M. V. Berry and K. E. Mount, "Semiclassical approximations in wave mechanics," Rep. Prog. Phys. 35, 315-397 (1972).

52 D. L. Kauffman, I. Kosztin, and K. Schulten, "Expansion method for stationary states of quantum billiards," Am. J. Phys. 67, 133-141 (1999). The authors use the exact results for the equilateral triangle as a benchmark to test their methods.

53 For a review of the connection between the Coulomb and 3D oscillator problems in classical and quantum mechanics, see A. K. Grant and J. L. Rosner, "Classical orbits in power law 
potentials," Am. J. Phys. 62, 310-315 (1994).

54 Y. Zarmi, "The Bertrand theorem revisited," Am. J. Phys. 70, 446-449 (2002).

55 D. S. Saxon, Elementary Quantum Mechanics (McGraw-Hill, NY, 1968), p. 182. 


\section{Figure Captions}

Fig. 1. Plots of the expansion coefficients, $\sum_{n=1}^{\infty}\left|a_{n}\right|^{2}$ (bottom), and the average energy, $\sum_{n=1}^{\infty} E_{n}\left|a_{n}\right|^{2}$ (middle), for zero-momentum Gaussian wave packets in the 1D infinite well, as the central position, $x_{0}$, is varied from the center $\left(x_{0} / a=1 / 2\right)$ to beyond the edge of the well $\left(x_{0} / a>1\right)$. The expression in Eq. (24) is used and contributions from the 40 lowest-lying states are included. The solid (dashed) curves correspond to $\Delta x_{0}=0.05(0.1)$. As expected, the normalization decreases uniformly from unity as more of the initial wave packet is outside the well. The average energy, however, increases as the initial wave packet is placed near the edge of the well, as the expansion in eigenstates attempts to reproduce the sharp discontinuity at the wall (as shown for the $x_{0}=0.95$ packet at the top.)

Fig. 2. Plots of the autocorrelation function, $|A(t)|^{2}$ versus $t$, over one revival time for zeromomentum $\left(p_{0}=0\right)$ Gaussian wave packets in the 1D infinite well. Plots for different initial position values, $x_{0}$, from the center $\left(x_{0} / a=0.5\right)$ to near one edge $\left(x_{0} / a=0.8\right)$ are shown. Exact revivals at $T_{\text {rev }}=4 \mu a^{2} / \hbar \pi$ are obvious for all initial positions, but special exact revivals at shorter times due to obvious symmetries in the eigenstate expansion at $x_{0}=a / 2$ (for multiples of $T_{\text {rev }} / 8$, one of which is highlighted in the rectangular area) and at $x_{0}=2 a / 3$ (for multiples of $T_{\text {rev }} / 3$, highlighted in the two elliptical areas) are also apparent. We note that there are partial revivals for $x_{0} / a=0.8$ (top line) for times given by $0.4 T_{\text {rev }}$ and $0.6 T_{\text {rev }}$ (shown by the diamonds.)

Fig. 3. Plots of the autocorrelation function, $|A(t)|^{2}$ versus $t$ (left column), over one revival time for $x_{0}=a / 2$ wave packets with increasing values of average momentum, $p_{0}$. For the bottom case $(\mathrm{d})$, the classical periodicity is so short that the individual periods cannot be resolved, in contrast to case (c) where the periods are clearly visible. Locations of fractional (partial) revivals are indicated by arrows for case (d) where they are most obvious. The corresponding values of the expansion coefficients, $\left|a_{n}\right|^{2}$ versus $n$, are shown on the right.

Fig. 4. Plots of the autocorrelation function, $|A(t)|^{2}$ versus $t$, for the square billiard. The plots are over a time period to ten classical back-and-forth periods, $10 \tau$, where $\tau=2 L / v_{0}$. 
Plots for different values of the initial angle, $\tan (\theta)=p_{0 y} / p_{0 x}$ are shown. The stars indicate the positions of classical closed orbits (and recurrences) as shown in Table I.

Fig. 5. Values of the (numerically evaluated) zeros of the Bessel function, $J_{|m|}(z=k a)$ as the quantum number, $m$, which determine the energy eigenvalues for the circular well, is varied. The (roughly elliptical) shaded regions correspond to regions in $\left(m, z=k_{m, n_{r}}\right)$ space inside which $68 \%$ (inner) and $99.7 \%$ of the $\sum_{\left(m, n_{r}\right)}\left|a_{\left(m, n_{r}\right)}\right|^{2}$ is contained. The three cases shown correspond to $y_{0}, p_{0 x}=0, p_{0 y}=100$ and $x_{0}=-0.5,0.0$, and 0.7 for Gaussian wave packets with the physical parameters in Eq. (25). The common value of $p_{0 y}=\hbar k=100$ is shown as a horizontal dashed line. (The faint vertical banding is an artifact of our plotting program.)

Fig. 6. Plots of the autocorrelation function, $|A(t)|^{2}$ versus $t$, for the circular billiard. The plots are for $x_{0}, p_{0 y} \neq 0$ with $y_{0}=p_{0 x}=0$ as one increases $x_{0} / R=R_{\min } / R$. The stars indicate the locations of classical closed orbits with periods and $R_{\min } / R$ values given by Eqs. (80) and (79), respectively as shown in Table II. Examples of closed orbit trajectories for several simple $(p, q)$ combinations are also shown at the corresponding values of $R_{\min } / R$. 


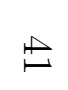
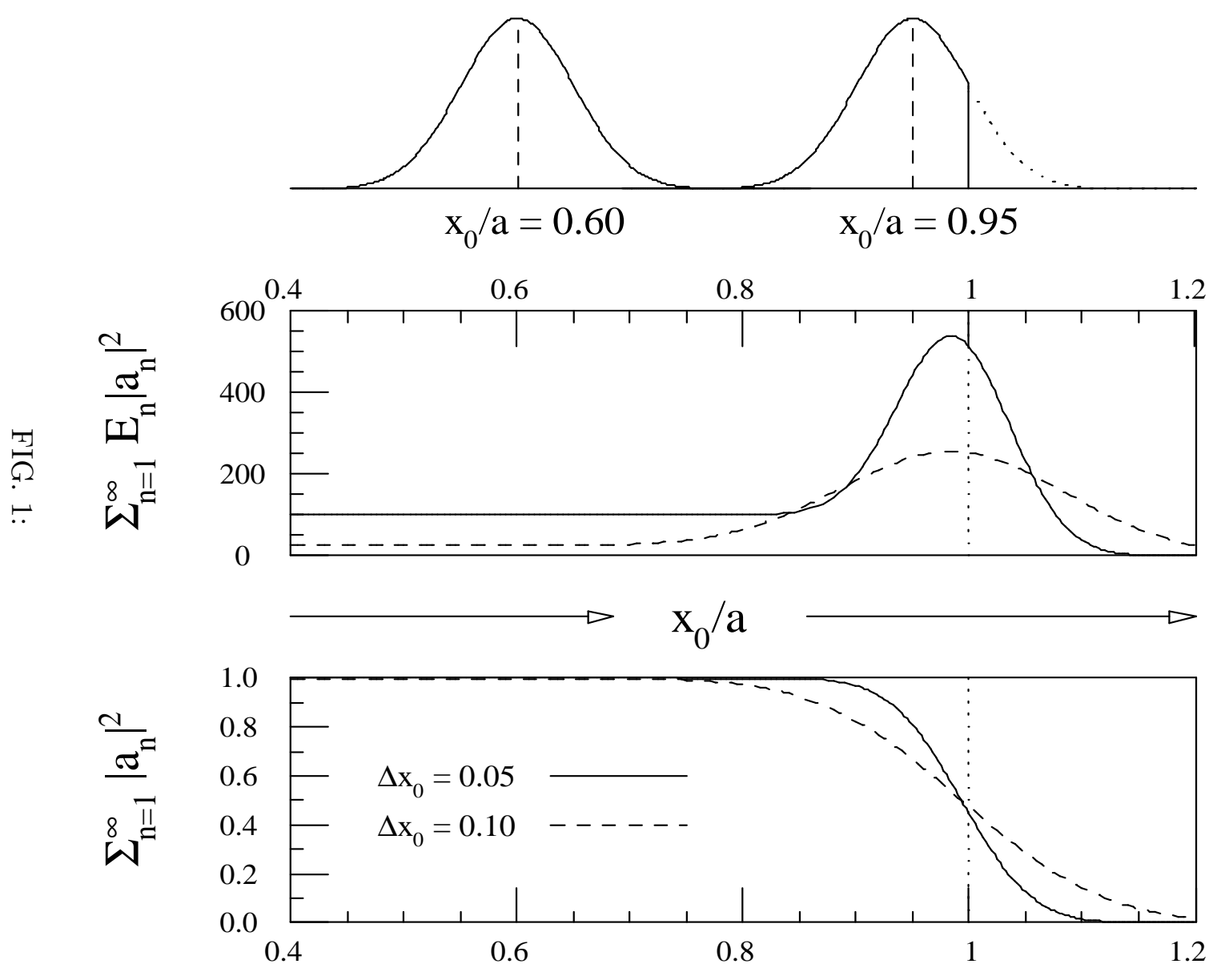


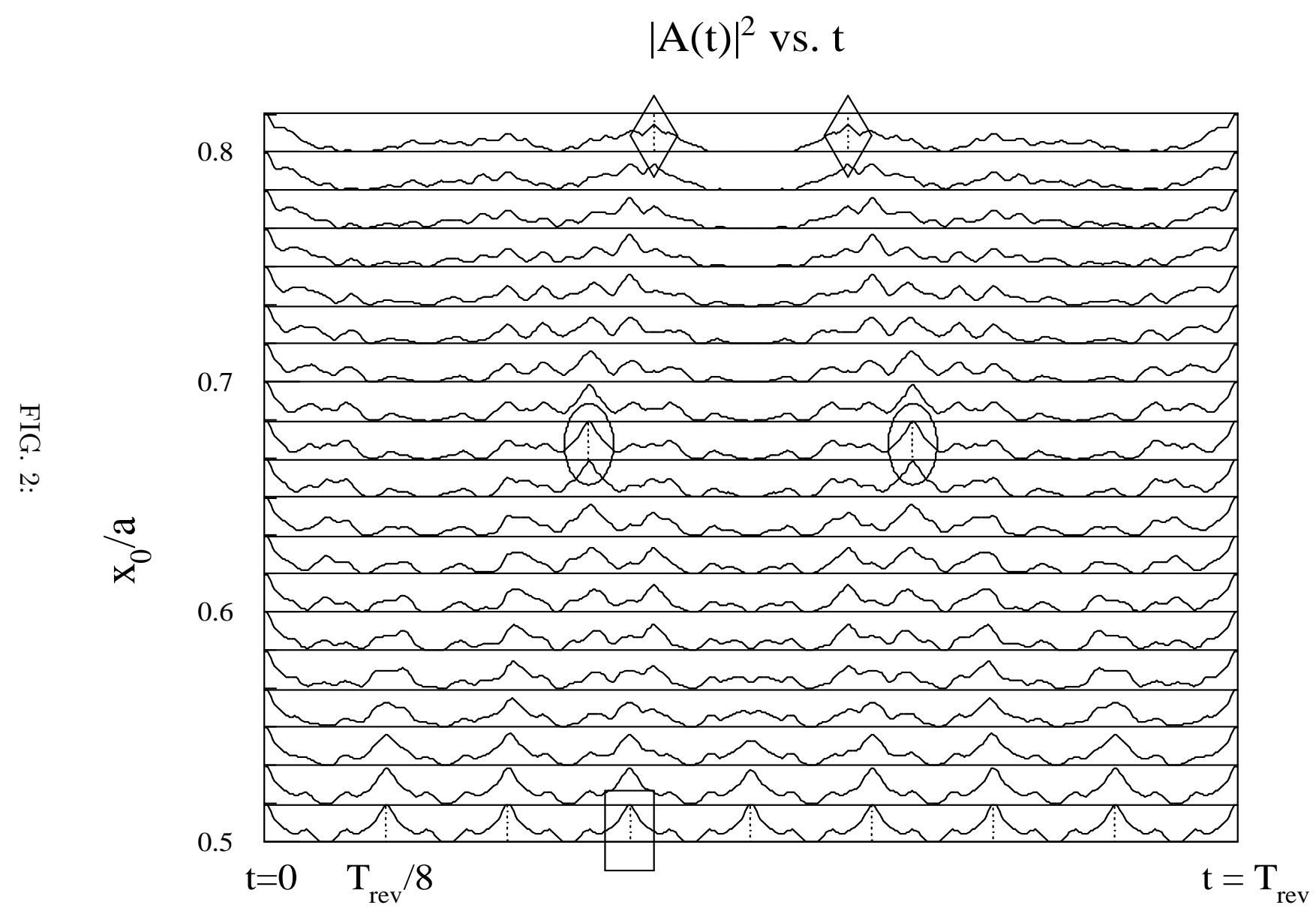



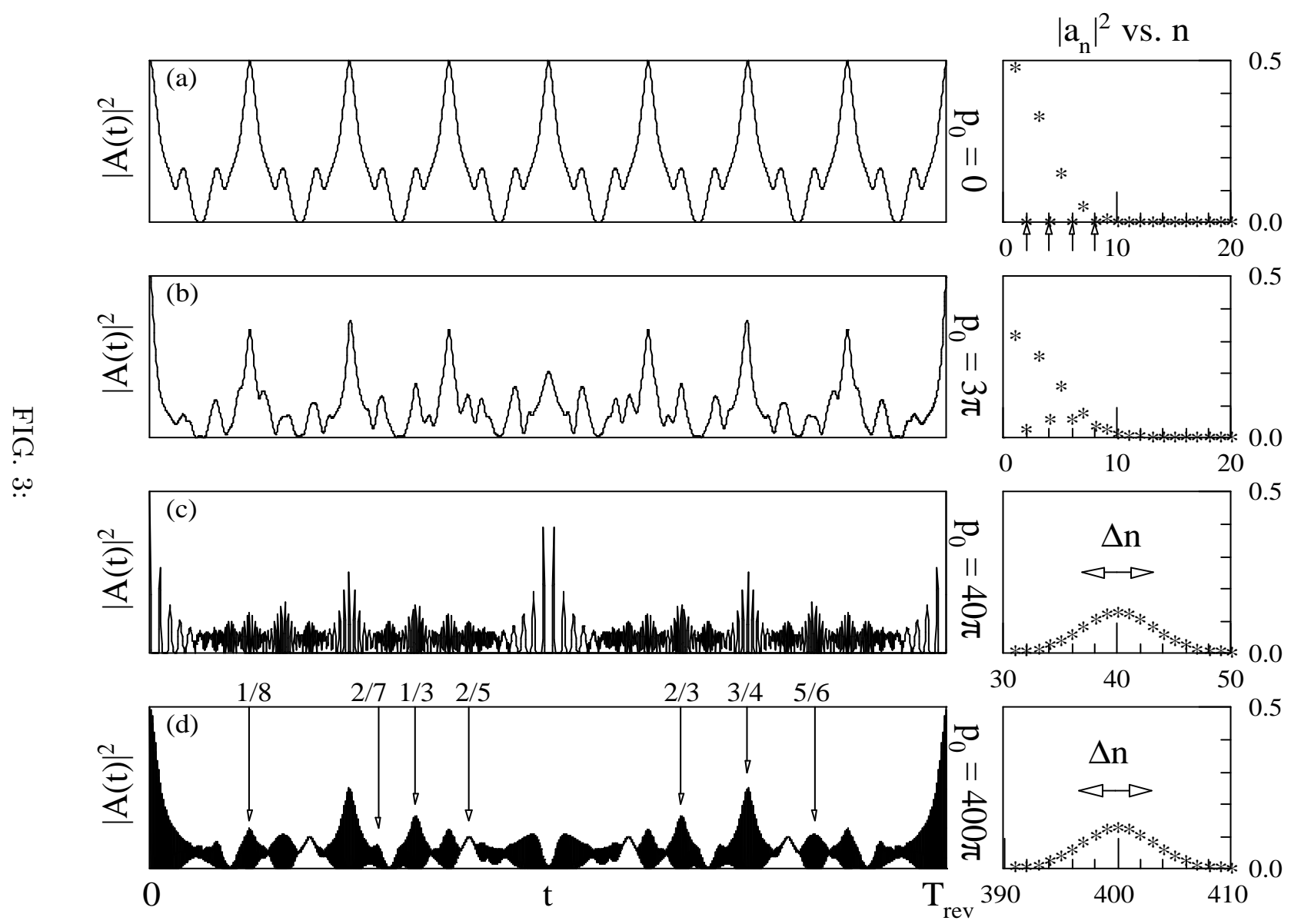


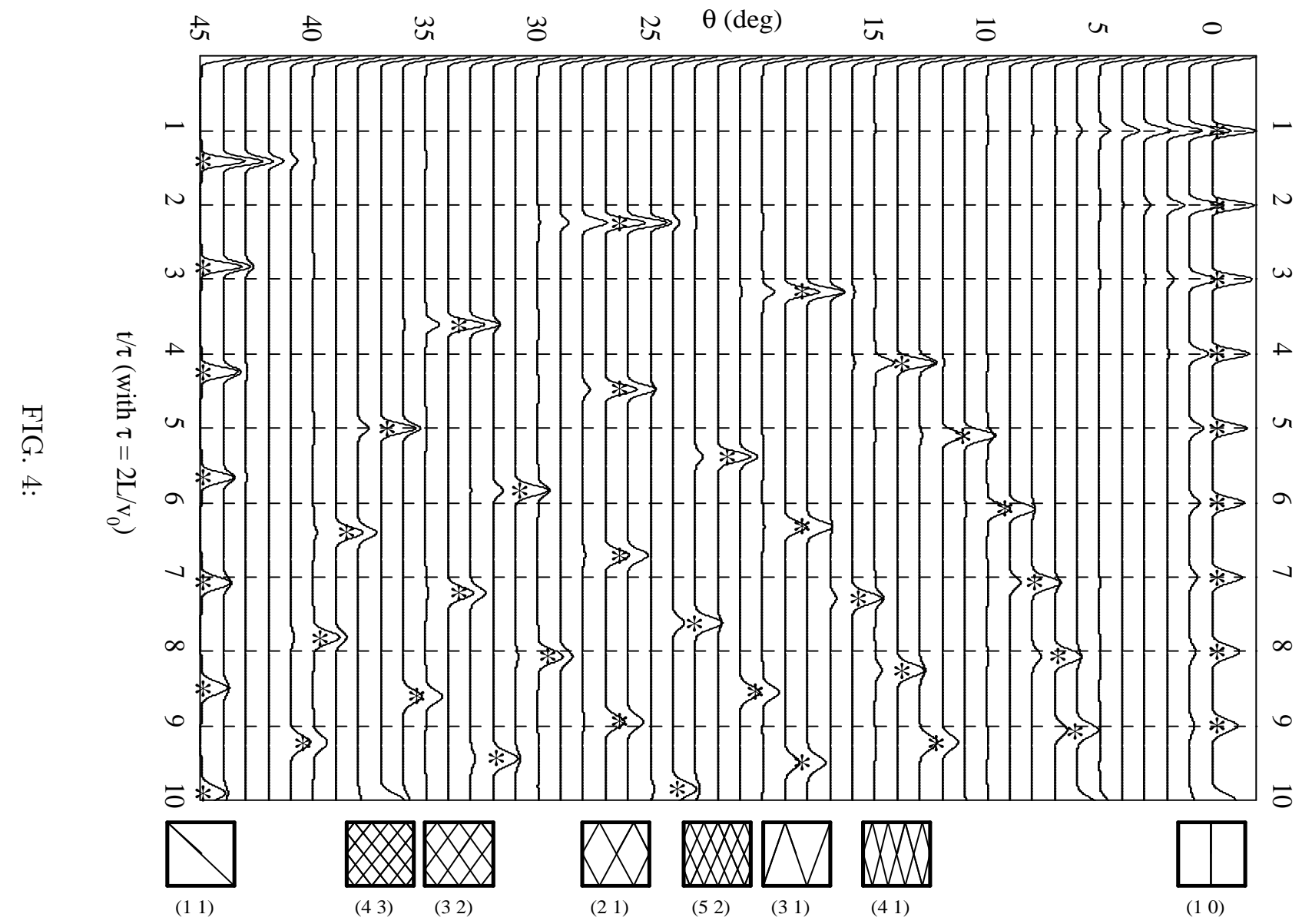




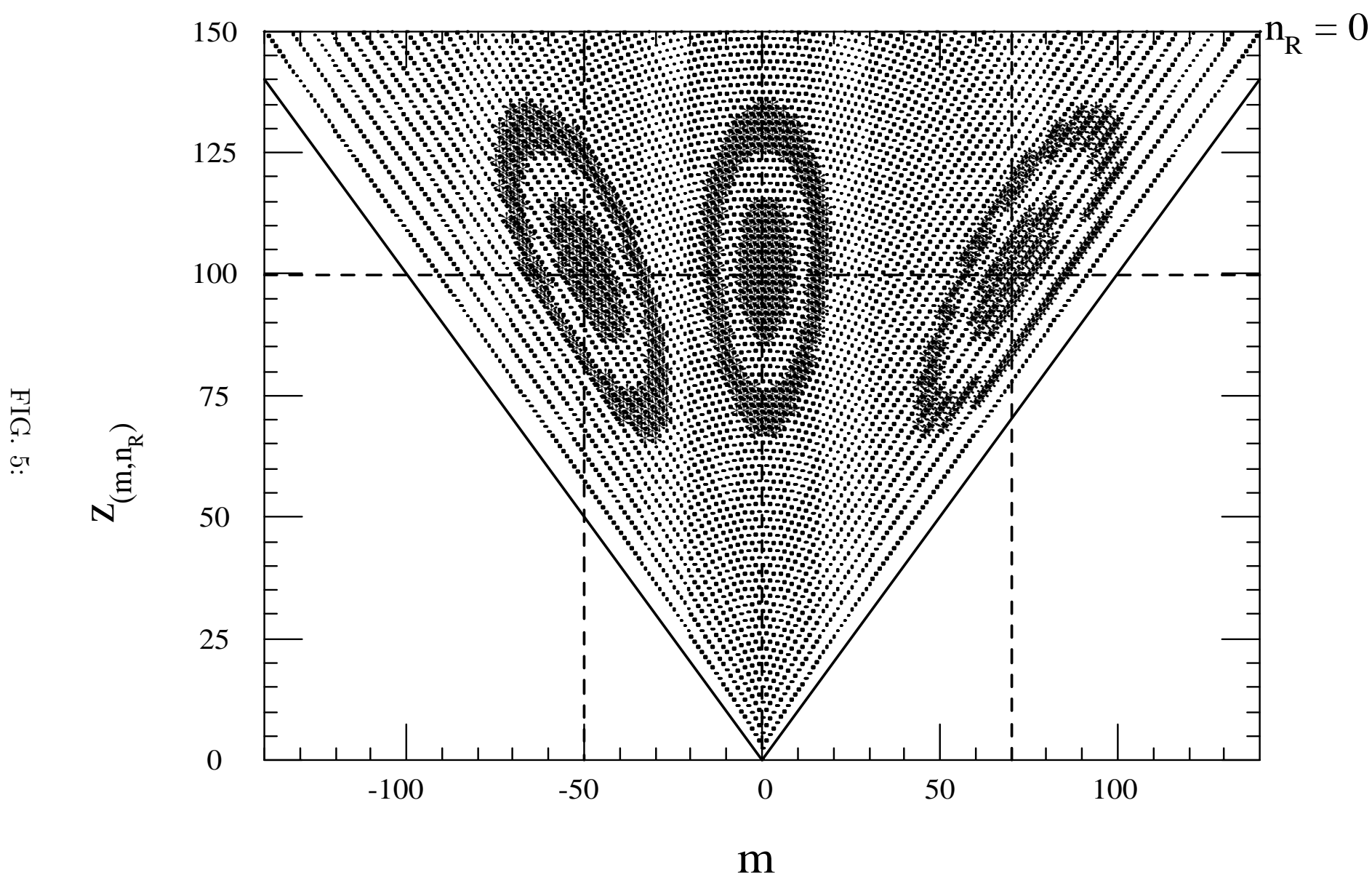




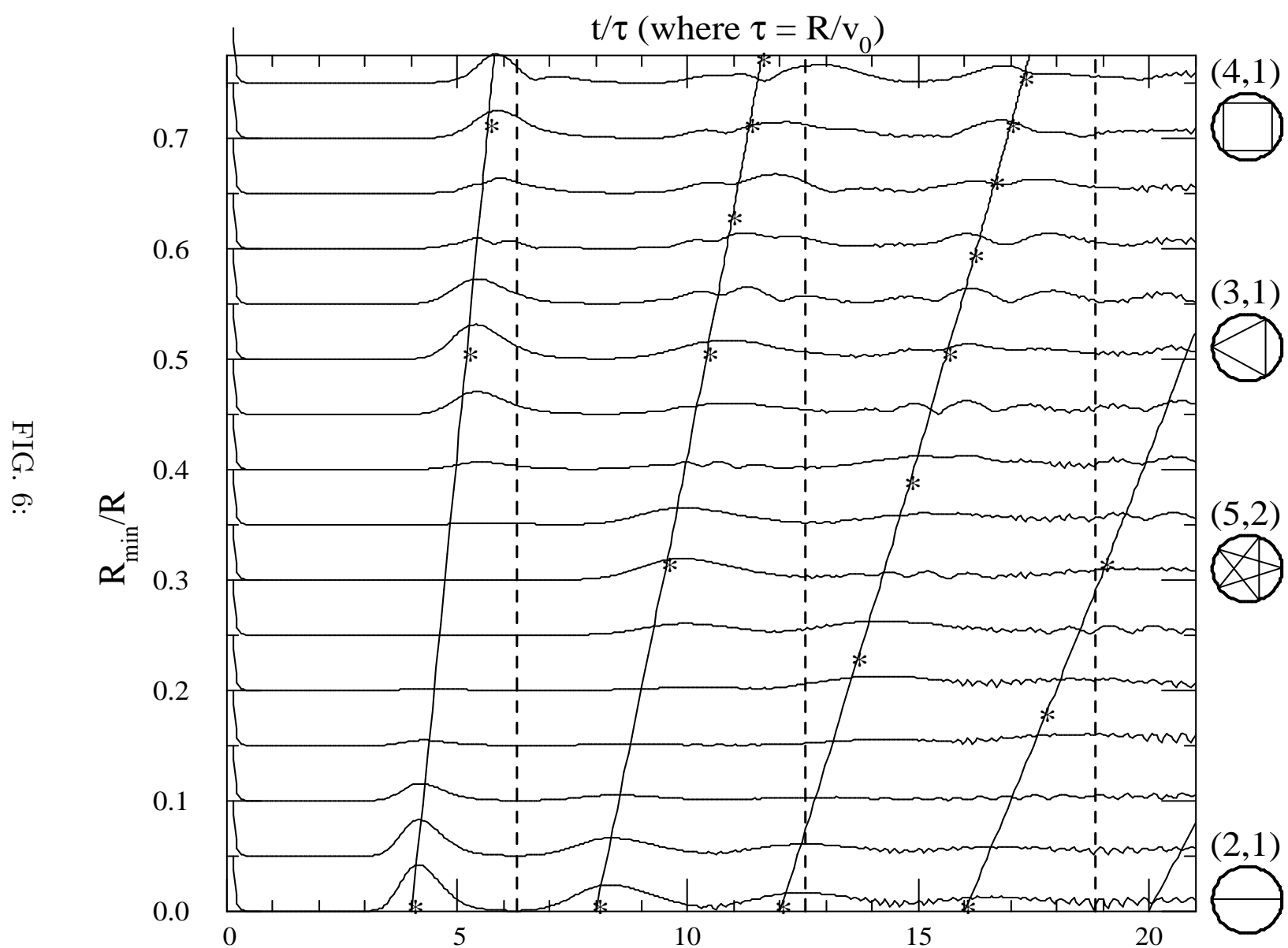

